\title{
ON THE OPTIMAL DIVIDEND STRATEGY IN A REGIME-SWITCHING DIFFUSION MODEL
}

\author{
JIAQIN WEI ${ }^{* * *}$ AND \\ RONGMING WANG, ${ }^{* * *}$ East China Normal University \\ HAILIANG YANG, ${ }^{* * * *}$ The University of Hong Kong
}

\begin{abstract}
In this paper we consider the optimal dividend strategy under the diffusion model with regime switching. In contrast to the classical risk theory, the dividends can only be paid at the arrival times of a Poisson process. By solving an auxiliary optimal problem we show that the optimal strategy is the modulated barrier strategy. The value function can be obtained by iteration or by solving the system of differential equations. We also provide a numerical example to illustrate the effects of the restriction on the timing of the payment of dividends.
\end{abstract}

Keywords: Dividend strategy; regime switching; HJB equation; Markov decision process 2010 Mathematics Subject Classification: Primary 93E20

Secondary 91B70; 60H30

\section{Introduction}

Since it was proposed by [7], the optimization of dividend strategy has become a classical and important problem in actuarial science. This problem is usually phrased as the management's problem of determining the optimal timing and size of dividend payments in the presence of bankruptcy risk. There is a vast literature on this topic. In most it is assumed that the insurer can choose any time to pay the dividends, or the dividends can be paid continuously, and the ruin (stopping the business) occurs whenever the surplus is negative. However, in practice, it is more reasonable that the dividends can only be paid at some discrete time points rather than continuously, and an insurer with a negative surplus may continue his/her business as usual until bankruptcy takes place. To capture these features, Albrecher et al. [1], [2] assumed that the surplus process can only be observed at random times. Then ruin can only occur and the dividends can only be paid at these random discrete observation times. With the assumption that the surplus process is observed at the arrival times of a Poisson process, Albrecher et al. [1] showed that the optimal strategy is a band strategy if the surplus process is modeled by a general Lévy process, and the optimal strategy reduces to the barrier strategy if the surplus process is a diffusion or the compound Poisson model with exponential claims.

Recently, Albrecher et al. [3] proposed the gamma-omega model, which extends the diffusion model in two ways. First, if the surplus $x$ is negative, the probability of bankruptcy within $\mathrm{d} t$ time units is $\omega(x) \mathrm{d} t$, where $\omega(x)>0$ is the decreasing bankruptcy rate function defined

Received 13 September 2011; revision received 23 January 2012.

* Postal address: School of Finance and Statistics, East China Normal University, Shanghai, 200241, China.

** Email address: jiaqinwei@gmail.com

*** Email address: rmwang@ stat.ecnu.edu.cn

**** Postal address: Department of Statistics and Actuarial Science, The University of Hong Kong, Pokfulam Road, Hong Kong, China. Email address: hlyang@hku.hk 
on $(-\infty, 0]$. Second, the dividends can only be paid to the shareholders at the arrival times of a Poisson process with rate $\gamma>0$. They studied the optimal barrier strategy, and Wei and Wang [13] proved that the optimal barrier strategy they obtained is indeed the optimal strategy among all the admissible dividend strategies under the gamma-omega model.

In this paper we consider the diffusion model with regime switching. We mainly consider the case where the dividends can only be paid at the arrival times of a modulated Poisson process (a Cox process) as in [3], and ruin is still defined as in the classical risk theory, i.e. the company is ruined and has to go out of business whenever the surplus is negative. In [3] and our paper, the surplus processes are observed continuously, but we restrict ourselves to the case where the dividends can only be paid at some random discrete times. From this point of view, the problem considered in our paper is similar to [12].

Under the diffusion model with regime switching, the optimal dividend strategy was studied by Sotomayor and Cadenillas [11] and Jiang and Pistorius [8]. While Sotomayor and Cadenillas solved this problem with two regimes by the standard method, i.e. guessing a candidate optimal solution and then verifying its optimality, Jiang and Pistorius solved a general case by following a different method. They constructed the candidate value function by directly employing a dynamic programming equation, proved that the value function is the fixed point of a certain contraction operator which is given with the initial data, and derived an explicit iterative algorithm to calculate the value function, which decouples the different regimes such that at any stage one-dimensional control problems are solved. In contrast, to prove that the value function is the fixed point of a contraction operator, we modify the procedure in [8] by constructing a sequence of functions that converges to the value function. Then we study the functions of this sequence by an auxiliary optimal problem which depends on only one regime. With such a sequence, we do not need to find priori bounds for the value function (or the initial data of the contraction operator), which is required in [8]. The idea of introducing such a sequence was motivated by Bayraktar and Ludkovski [5] and Davis [6, pp. 188-204], who considered the optimal control problem under piecewise-deterministic processes. In fact, by this method, we reduce the original problem to a Markov decision process (MDP) (we thank the referee for pointing out this fact, as well as Remarks 3.1 and 3.2), which was also used in [1]. Similar to [8] and [11], our optimal strategy is still the modulated barrier strategy.

The remainder of the paper is organized as follows. In Section 2 we present the model and the problem. In Section 3 we introduce a sequence of functions that converges to the value function, and prove the dynamic programming equation. The original problem is also reduced to an MDP. In Section 4, in order to study the sequence constructed in Section 3, we study an auxiliary optimal problem which is the one-stage problem of the MDP. In Section 5 we return to our original optimal problem. We show two ways to obtain the value function and the optimal barrier levels.

\section{The model}

Suppose that $\{J(t)\}_{t \geq 0}$ is a homogeneous, irreducible, continuous-time Markov chain taking values in a finite set $\mathbb{J}=\{1,2, \ldots, K\}$ with generator $\boldsymbol{Q}=\left(q_{i j}\right)_{K \times K}$, where $-q_{i i}=q_{i}>0$ for $i \in \mathbb{J}$. Let $X_{i}(t)=\mu_{i} t+\sigma_{i} W(t)$, where $\mu_{i}, \sigma_{i}>0$ for all $i \in \mathbb{J}$, and $\{W(t)\}_{t \geq 0}$ is a standard Brownian motion which is independent of $\{J(t)\}_{t \geq 0}$. The surplus process of the insurer is given by

$$
X(t)=x+\sum_{i=1}^{K} \int_{0}^{t} \mathbf{1}_{\{J(s)=i\}} \mathrm{d} X_{i}(s),
$$

where $x>0$ is the initial surplus. 
When the state of the Markov chain is $i \in \mathbb{J}$, we assume that the dividends can only be paid at the arrival times of a Poisson process with rate $\gamma_{i}>0$. Considering dividends, the surplus process (still denoted by $\{X(t)\}_{t \geq 0}$ ) is given by

$$
X(t)=x+\sum_{i=1}^{K} \int_{0}^{t} \mathbf{1}_{\{J(s)=i\}} \mathrm{d} X_{i}(s)-D(t),
$$

where $D(t)$ is the cumulative dividends until $t$. Let $\left\{N_{i}(t)\right\}_{t \geq 0}$ be a Poisson process with intensity $\gamma_{i}$ which is assumed to be independent of $\{J(t)\}_{t \geq 0}$ and $\{W(t)\}_{t \geq 0}$. Then we can write

$$
D(t)=\sum_{i=1}^{K} \int_{0}^{t} \pi(s) \mathbf{1}_{\{J(s)=i\}} \mathrm{d} N_{i}(s),
$$

where the process $\{\pi(s)\}_{s \geq 0}$ determines the amount of dividends paid at the jump times of the Poisson processes $\left\{N_{i}(t)\right\}_{t \geq 0}, i \in \mathbb{J}$.

Suppose that all the stochastic processes mentioned above are defined on the filtered probability space $(\Omega, \mathcal{F}, \mathrm{P})$, where $\mathcal{F}=\left\{\mathcal{F}_{t}, t \geq 0\right\}$ is generated by $\{X(t)\}_{t \geq 0}$ and $\{J(t)\}_{t \geq 0}$ and satisfies the usual conditions. Denote by $\mathrm{E}_{x}$ and $\mathrm{E}_{x, i}$ the expectations conditioned on $\{X(0)=x\}$ and $\{X(0)=x, J(0)=i\}$, respectively.

We say that a dividend strategy $\{\pi(s)\}_{s \geq 0}$ (for convenience, we also write $\pi$ for short) is admissible if it is $\mathcal{F}$-adapted and $0 \leq \pi(t)<X(t-)$ for $t \geq 0$. Let $\Pi$ be the set of all admissible strategies. With a strategy $\pi \in \Pi$, let $\tau_{\pi}:=\inf \{t \geq 0: X(t) \leq 0\}$ be the time of ruin. Without loss of generality, we assume that $X(t) \equiv 0$ for $t \geq \tau_{\pi}$. Given the initial surplus $x$ and initial state $i$, the expected value of the discounted dividends until ruin is given by

$$
V_{\pi}(x, i):=\mathrm{E}_{x, i}\left[\sum_{k=1}^{K} \int_{0}^{\tau_{\pi}} \mathrm{e}^{-\Lambda(s)} \mathbf{1}_{\{J(s)=k\}} \pi(s) \mathrm{d} N_{k}(s)\right],
$$

where $\Lambda(s)=\sum_{i=1}^{K} \int_{0}^{s} \mathbf{1}_{\{J(t)=i\}} \delta_{i} \mathrm{~d} t$, with $\delta_{i}>0$, is the discount rate at state $i$ for $i \in \mathbb{J}$. The objective function is

$$
V(x, i)=\sup _{\pi \in \Pi} V_{\pi}(x, i), \quad i=1,2, \ldots, K .
$$

It is easy to see that $V(0, i)=0$ for all $i \in \mathbb{J}$. The problem of the shareholders is to specify a dividend strategy $\pi^{*} \in \Pi$ such that $V(x, i)=V_{\pi^{*}}(x, i)$ for all $i \in \mathbb{J}$.

\section{The dynamic programming equation}

In the following, we adopt bold letters to denote vector functions of the form

$$
\boldsymbol{v}(x):=(v(x, 1), v(x, 2), \ldots, v(x, K)) .
$$

When we use ' $\leq$ ' or ' $\geq$ ' between two vectors (or vector functions), it respectively means that ' $\leq$ ' or ' $\geq$ ' holds for each element. Also, we denote by $\mathbf{0}$ the zero row vector with $K$ elements.

Let $\zeta_{0}=0$ and

$$
\zeta_{n}:=\inf \left\{t \geq \zeta_{n-1}: J(t) \neq J(t-)\right\}, \quad n \in \mathbb{N}^{+},
$$


i.e. $\zeta_{n}$ is the $n$th jump time of the Markov chain $\{J(t)\}_{t \geq 0}$. For a test function $v(x)$, define the functional operator as

$$
\mathcal{M} \boldsymbol{v}(x):=(\mathcal{M} v(x, 1), \mathcal{M} v(x, 2), \ldots, \mathcal{M} v(x, K))
$$

where

$$
\mathcal{M} v(x, i):=\sup _{\pi \in \Pi} \mathrm{E}_{x, i}\left[\int_{0}^{\tau_{\pi} \wedge \zeta_{1}} \mathrm{e}^{-\delta_{i} s} \pi(s) \mathrm{d} N_{i}(s)+\mathrm{e}^{-\delta_{i}\left(\tau_{\pi} \wedge \zeta_{1}\right)} v\left(X\left(\tau_{\pi} \wedge \zeta_{1}\right), J\left(\tau_{\pi} \wedge \zeta_{1}\right)\right)\right] .
$$

From the definition of $\mathcal{M}$, we have the following lemmas.

Lemma 3.1. If $\boldsymbol{v}_{1}(x) \geq \boldsymbol{v}_{2}(x)$ then it holds that $\mathcal{M} \boldsymbol{v}_{1}(x) \geq \mathcal{M} \boldsymbol{v}_{2}(x)$ for all $x \geq 0$.

Lemma 3.2. For all $x \geq 0$, let $\boldsymbol{U}_{0}(x) \equiv \mathbf{0}$ and $\boldsymbol{U}_{n+1}(x)=\mathcal{M} \boldsymbol{U}_{n}(x)$ for $n \in \mathbb{N}$. Then, for each $i \in \mathbb{J},\left\{U_{n}(\cdot, i)\right\}_{n \in \mathbb{N}}$ is an increasing sequence of functions.

Proof. Note that $U_{1}(x, i)=\sup _{\pi \in \Pi} \mathrm{E}_{x, i}\left[\int_{0}^{\tau_{\pi} \wedge \zeta_{1}} \mathrm{e}^{-\delta_{i} s} \pi(s) \mathrm{d} N_{i}(s)\right] \geq 0=U_{0}(x, i)$ for all $x \geq 0$ and $i \in \mathbb{J}$. The result follows from Lemma 3.1.

For $n \in \mathbb{N}$, define $\Pi_{n}=\left\{\pi \in \Pi: \pi(s) \equiv 0\right.$ for $\left.s \geq \zeta_{n}\right\}$ to be the set of all the admissible strategies that pays no dividend after the $n$th jump of the Markov chain $\{J(t)\}_{t \geq 0}$. Let $V_{n}(x, i)=$ $\sup _{\pi \in \Pi_{n}} V_{\pi}(x, i)$.

Lemma 3.3. For all $x \geq 0$, we have $\boldsymbol{V}_{n}(x)=\boldsymbol{U}_{n}(x)$ for all $n \in \mathbb{N}$.

Proof. Obviously, we have $\boldsymbol{V}_{0}(x)=\boldsymbol{U}_{0}(x) \equiv \mathbf{0}$. Let us assume that $\boldsymbol{V}_{n}(x)=\boldsymbol{U}_{n}(x)$, and show that $\boldsymbol{V}_{n+1}(x)=\boldsymbol{U}_{n+1}(x)$.

First, we will show that $\boldsymbol{V}_{n+1}(x) \leq \boldsymbol{U}_{n+1}(x)$. For any $\varepsilon>0$, there exists a strategy $\pi \in \Pi_{n+1}$ such that

$$
V_{\pi}(x, i) \geq V_{n+1}(x, i)-\varepsilon .
$$

Define a strategy $\hat{\pi} \in \Pi_{n}$ by setting $\hat{\pi}(t)=\pi\left(t+\tau_{\pi} \wedge \zeta_{1}\right)$ for $t \geq 0$. By the strong Markov property we have

$$
\begin{aligned}
V_{\pi}(x, i) & =\mathrm{E}_{x, i}\left[\sum_{k=1}^{K} \int_{0}^{\tau_{\pi}} \mathrm{e}^{-\Lambda(s)} \pi(s) \mathbf{1}_{\{J(s)=k\}} \mathrm{d} N_{k}(s)\right] \\
& =\mathrm{E}_{x, i}\left[\int_{0}^{\tau_{\pi} \wedge \zeta_{1}} \mathrm{e}^{-\delta_{i} s} \pi(s) \mathrm{d} N_{i}(s)+\mathrm{e}^{-\delta_{i}\left(\tau_{\pi} \wedge \zeta_{1}\right)} V_{\hat{\pi}}\left(X\left(\tau_{\pi} \wedge \zeta_{1}\right), J\left(\tau_{\pi} \wedge \zeta_{1}\right)\right)\right] \\
& \leq \mathrm{E}_{x, i}\left[\int_{0}^{\tau_{\pi} \wedge \zeta_{1}} \mathrm{e}^{-\delta_{i} s} \pi(s) \mathrm{d} N_{i}(s)+\mathrm{e}^{-\delta_{i}\left(\tau_{\pi} \wedge \zeta_{1}\right)} V_{n}\left(X\left(\tau_{\pi} \wedge \zeta_{1}\right), J\left(\tau_{\pi} \wedge \zeta_{1}\right)\right)\right] \\
& =\mathrm{E}_{x, i}\left[\int_{0}^{\tau_{\pi} \wedge \zeta_{1}} \mathrm{e}^{-\delta_{i} s} \pi(s) \mathrm{d} N_{i}(s)+\mathrm{e}^{-\delta_{i}\left(\tau_{\pi} \wedge \zeta_{1}\right)} U_{n}\left(X\left(\tau_{\pi} \wedge \zeta_{1}\right), J\left(\tau_{\pi} \wedge \zeta_{1}\right)\right)\right] \\
& \leq U_{n+1}(x, i) .
\end{aligned}
$$

It follows, from (3.1), (3.2), and the arbitrariness of $\varepsilon$, that $V_{n+1}(x, i) \leq U_{n+1}(x, i)$ for all $x \geq 0$ and $i \in \mathbb{J}$. 
Second, we will show that $\boldsymbol{V}_{n+1}(x) \geq \boldsymbol{U}_{n+1}(x)$. For any $\varepsilon>0$, there exists a strategy $\pi^{\prime} \in \Pi$ such that

$$
\begin{aligned}
U_{n+1}(x, i) \leq & \mathrm{E}_{x, i}\left[\int_{0}^{\tau_{\pi^{\prime}} \wedge \zeta_{1}} \mathrm{e}^{-\delta_{i} s} \pi^{\prime}(s) \mathrm{d} N_{i}(s)+\mathrm{e}^{-\delta_{i}\left(\tau_{\pi^{\prime}} \wedge \zeta_{1}\right)} U_{n}\left(X\left(\tau_{\pi^{\prime}} \wedge \zeta_{1}\right), J\left(\tau_{\pi^{\prime}} \wedge \zeta_{1}\right)\right)\right] \\
& +\varepsilon
\end{aligned}
$$

and there exists a strategy $\pi^{\prime \prime} \in \Pi_{n}$ such that $V_{n}(x, i) \leq V_{\pi_{2}}(x, i)$ for any $x \geq 0$ and $i \in \mathbb{J}$. Now we can construct a strategy $\tilde{\pi} \in \Pi_{n+1}$ by taking the strategy $\pi^{\prime}$ before $\tau_{\pi^{\prime}} \wedge \zeta_{1}$, and then following strategy $\pi^{\prime \prime}$. Thus, by the strong Markov property we have

$$
\begin{aligned}
U_{n+1}(x, i) \leq & \mathrm{E}_{x, i}\left[\int_{0}^{\tau_{\pi^{\prime}} \wedge \zeta_{1}} \mathrm{e}^{-\delta_{i} s} \pi^{\prime}(s) \mathrm{d} N_{i}(s)+\mathrm{e}^{-\delta_{i}\left(\tau_{\pi^{\prime}} \wedge \zeta_{1}\right)} V_{n}\left(X\left(\tau_{\pi^{\prime}} \wedge \zeta_{1}\right), J\left(\tau_{\pi^{\prime}} \wedge \zeta_{1}\right)\right)\right] \\
& +\varepsilon \\
\leq & \mathrm{E}_{x, i}\left[\int_{0}^{\tau_{\pi^{\prime}} \wedge \zeta_{1}} \mathrm{e}^{-\delta_{i} s} \pi^{\prime}(s) \mathrm{d} N_{i}(s)+\mathrm{e}^{-\delta_{i}\left(\tau_{\pi^{\prime}} \wedge \zeta_{1}\right)} V_{\pi^{\prime \prime}}\left(X\left(\tau_{\pi^{\prime}} \wedge \zeta_{1}\right), J\left(\tau_{\pi^{\prime}} \wedge \zeta_{1}\right)\right)\right] \\
& +2 \varepsilon \\
= & V_{\tilde{\pi}}(x, i)+2 \varepsilon \\
\leq & V_{n+1}(x, i)+2 \varepsilon .
\end{aligned}
$$

Thus, from the arbitrariness of $\varepsilon$, we have $U_{n+1}(x, i) \leq V_{n+1}(x, i)$ for all $x \geq 0$ and $i \in \mathbb{J}$, which completes our proof.

Remark 3.1. Note that $\mathcal{M}$ can be interpreted as an MDP operator of a positive MDP, and our original problem boils down to solving an MDP. The following results are standard (see, e.g. [4]).

Lemma 3.4. It holds that $\lim _{n \rightarrow \infty} U_{n}(x, i)=V(x, i)$ for any $x \geq 0$ and $i \in \mathbb{J}$.

Proposition 3.1. The value function $\boldsymbol{V}$ is the smallest solution of the dynamic programming equation $\boldsymbol{V}=\mathcal{M} \boldsymbol{V}$ such that $\boldsymbol{V} \geq \mathbf{0}$, i.e.

$$
V(x, i)=\sup _{\pi \in \Pi} \mathrm{E}_{x, i}\left[\int_{0}^{\tau_{\pi} \wedge \zeta_{1}} \mathrm{e}^{-\delta_{i} s} \pi(s) \mathrm{d} N_{i}(s)+\mathrm{e}^{-\delta_{i}\left(\tau_{\pi} \wedge \zeta_{1}\right)} V\left(X\left(\tau_{\pi} \wedge \zeta_{1}\right), J\left(\tau_{\pi} \wedge \zeta_{1}\right)\right)\right]
$$

for all $x \geq 0$ and $i \in \mathbb{J}$.

Remark 3.2. In general, for positive MDPs, it is not true that a maximizer of the right-hand side of (3.3) yields the optimal strategy. Let $\tilde{V}$ be the value function studied in [8]. Then there exists a constant $c>0$ such that $\tilde{V}(x, i)<x+c$ for all $i \in \mathbb{J}$. Note that the set of admissible strategies $\Pi$ in this paper is a subset of that considered in [8]. It follows that $V(x, i) \leq \tilde{V}(x, i)<x+c$ for all $x \in[0, \infty)$ and $i \in \mathbb{J}$. For $i \in \mathbb{J}$, define $b(x, i):=1+x$ and the operator

$$
\mathcal{T}_{o} v(x, i):=\sup _{\pi \in \Pi} \mathrm{E}_{x, i}\left[\mathrm{e}^{-\delta_{i}\left(\tau_{\pi} \wedge \zeta_{1}\right)} v\left(X\left(\tau_{\pi} \wedge \zeta_{1}\right), J\left(\tau_{\pi} \wedge \zeta_{1}\right)\right)\right]
$$

Considering a strategy $\pi \in \Pi$, let

$$
Y(t)=x+X_{i}(t)-\int_{0}^{t} \pi(s) \mathrm{d} N_{i}(s)
$$


and let $\tau_{i}$ be the time of ruin of $\{Y(t)\}_{t \geq 0}$. For any constant $\theta>0$, denote by $\eta(\theta)$ an independent exponential random variable with mean $1 / \theta$. It holds that $\left(Y(t), t<\tau_{i} \wedge \eta\left(q_{i}\right)\right)$ is in distribution equal to $\left(X(t), J(0)=i, t<\tau_{\pi} \wedge \zeta_{1}\right)$. It is easy to see that

$$
\begin{aligned}
\mathcal{T}_{o} b(x, i) & =\sup _{\pi \in \Pi} \mathrm{E}_{x}\left[\int_{0}^{\tau_{i}} \mathrm{e}^{-\left(\delta_{i}+q_{i}\right) s} \sum_{j \neq i} q_{i j} b(Y(s), j) \mathrm{d} s\right] \\
& \leq \mathrm{E}_{x}\left[\int_{0}^{\infty} \mathrm{e}^{-\left(\delta_{i}+q_{i}\right) s} q_{i}\left(1+x+\mu_{i} s+\sigma_{i} W(s)\right) \mathrm{d} s\right] \\
& =\frac{q_{i}}{\delta_{i}+q_{i}}\left(1+x+\frac{\mu_{i}}{\delta_{i}+\mu_{i}}\right) .
\end{aligned}
$$

Thus, by iteration we have $\lim _{n \rightarrow \infty} \mathcal{T}_{o}^{n} b(x, i)=0$, which implies that the maximizer of the right-hand side of (3.3) always gives the optimal strategy (see, e.g. [1] and [4, p. 209]). From the last equation we can see that $\delta_{i}>0$ is a crucial assumption.

\section{The solution to $U_{n}(x)$}

From Section 3 we know that the value function can be obtained by iteration. However, to do this, we need to show what $\boldsymbol{U}_{n+1}$ is when $\boldsymbol{U}_{n}$ is given. This is the problem studied in this section.

\subsection{An auxiliary optimal problem}

To solve our problem, we restrict ourselves to a special class of vector functions.

Definition 4.1. We say that a vector function $\boldsymbol{u}(x) \in \mathbb{D}$ if

(i) $\boldsymbol{u}(0)=\mathbf{0}$, and $u(\cdot, i) \in C([0, \infty))$ is increasing and concave for each $i \in \mathbb{J}$;

(ii) for any $\theta>0, \lim _{x \rightarrow \infty} \mathrm{e}^{-\theta x} u(x, i)=0$ for each $i \in \mathbb{J}$.

For a function $\boldsymbol{u} \in \mathbb{D}$, we consider the auxiliary optimal problem

$$
M(x, i):=\sup _{\pi \in \Pi} M_{\pi}(x, i)
$$

where

$$
M_{\pi}(x, i)=\mathrm{E}_{x, i}\left[\int_{0}^{\tau_{\pi} \wedge \zeta_{1}} \mathrm{e}^{-\delta_{i} s} \pi(s) \mathrm{d} N_{i}(s)+\mathrm{e}^{-\delta_{i}\left(\tau_{\pi} \wedge \zeta_{1}\right)} u\left(X\left(\tau_{\pi} \wedge \zeta_{1}\right), J\left(\tau_{\pi} \wedge \zeta_{1}\right)\right)\right] .
$$

From the general theory of stochastic control, we consider the Hamilton-Jacob-Bellman (HJB) equation

$$
\begin{gathered}
\max _{0 \leq a \leq x}\left\{\frac{\sigma_{i}^{2}}{2} m^{\prime \prime}(x, i)+\mu_{i} m^{\prime}(x, i)-\left(\delta_{i}+q_{i}+\gamma_{i}\right) m(x, i)\right. \\
\left.+\gamma_{i}[m(x-a, i)+a]+\sum_{j \neq i} q_{i j} u(x, j)\right\}=0
\end{gathered}
$$

for the optimal problem (4.1), where $m^{\prime}$ and $m^{\prime \prime}$ are the first- and second-order partial derivatives with respect to $x$, respectively. 
Theorem 4.1. For $i \in \mathbb{J}$, let $m(\cdot, i) \in C^{2}([0, \infty))$ be a nonnegative function. Assume that $m(x, i)$ satisfies the HJB equation (4.2) for all $x \geq 0$. Then

(i) $m(x, i) \geq M(x, i)$ for all $x \geq 0$;

(ii) if, in addition, $m(x, i)=M_{\pi^{*}}(x, i)$ for some $\pi^{*} \in \Pi$, $\pi^{*}$ is an optimal dividend strategy for problem (4.1) and $M(x, i) \equiv M_{\pi^{*}}(x, i)$.

Proof. (i) Considering a strategy $\pi \in \Pi$ and recalling $\{Y(t)\}_{t \geq 0}$ defined in (3.4), for any $\boldsymbol{u} \in \mathbb{D}$, we have

$$
\begin{aligned}
M_{\pi}(x, i)=\sup _{\pi \in \Pi} \mathrm{E}_{x}\left[\int_{0}^{\tau_{i}} \mathbf{1}_{\left\{s<\eta\left(q_{i}\right)\right\}} \mathrm{e}^{-\delta_{i} s} \pi(s) \mathrm{d} N_{i}(s)\right. \\
\left.+\mathbf{1}_{\left\{\eta\left(q_{i}\right)<\tau_{i}\right\}} \mathrm{e}^{-\delta_{i} \eta\left(q_{i}\right)} \sum_{j \neq i} \frac{q_{i j}}{q_{i}} u\left(Y\left(\eta\left(q_{i}\right)\right), j\right)\right] \\
=\sup _{\pi \in \Pi} \mathrm{E}_{x}\left[\int_{0}^{\tau_{i}} \mathrm{e}^{-\left(\delta_{i}+q_{i}\right) s} \pi(s) \mathrm{d} t N_{i}(s)+\int_{0}^{\tau_{i}} \mathrm{e}^{-\left(\delta_{i}+q_{i}\right) s} \sum_{j \neq i} q_{i j} u(Y(s), j) \mathrm{d} s\right] .
\end{aligned}
$$

Let $a$ and $b$ be real numbers satisfying $0<a<Y(0)=x<b<\infty$. Define $\tau_{a}:=\inf \{t \geq$ $0: Y(t) \leq a\}, \tau_{b}:=\inf \{t \geq 0: Y(t) \geq b\}$, and $\tau_{a b}=\tau_{a} \wedge \tau_{b}$. Applying the Itô formula to $\mathrm{e}^{-\delta_{i} t} m(Y(t), i)$ yields

$$
\begin{aligned}
& \mathrm{e}^{-\left(\delta_{i}+q_{i}\right)\left(t \wedge \tau_{a b}\right)} m\left(Y\left(t \wedge \tau_{a b}\right), i\right)-m(Y(0), i) \\
& =\int_{0}^{t \wedge \tau_{a b}} \mathrm{e}^{-\left(\delta_{i}+q_{i}\right) s}\left[-\left(\delta_{i}+q_{i}\right) m(Y(s), i)+\mu_{i} m^{\prime}(Y(s), i)+\frac{1}{2} \sigma_{i}^{2} m^{\prime \prime}(Y(s), i)\right] \mathrm{d} s \\
& \quad+\int_{0}^{t \wedge \tau_{a b}} \mathrm{e}^{-\left(\delta_{i}+q_{i}\right) s}[m(Y(s-)-\pi(s), i)-m(Y(s-), i)] \mathrm{d} N_{i}(s) \\
& \quad+\int_{0}^{t \wedge \tau_{a b}} \mathrm{e}^{-\left(\delta_{i}+q_{i}\right) s} \sigma_{i} m^{\prime}(Y(s), i) \mathrm{d} W(s) \quad \text { for all } t \geq 0 .
\end{aligned}
$$

Since $m(\cdot, i)$ satisfies $(4.2)$, we have

$$
\begin{aligned}
& \int_{0}^{t \wedge \tau_{a b}} \mathrm{e}^{-\left(\delta_{i}+q_{i}\right) s} \pi(s) \mathrm{d} N_{i}(s)+\int_{0}^{t \wedge \tau_{a b}} \mathrm{e}^{-\left(\delta_{i}+q_{i}\right) s} \sum_{j \neq i} q_{i j} u(Y(s), j) \mathrm{d} s \\
& \quad \leq-\mathrm{e}^{-\left(\delta_{i}+q_{i}\right)\left(t \wedge \tau_{a b}\right)} m\left(Y\left(t \wedge \tau_{a b}\right), i\right)+m(Y(0), i)+Z_{1}\left(t \wedge \tau_{a b}\right)+Z_{2}\left(t \wedge \tau_{a b}\right),
\end{aligned}
$$

where $\left\{Z_{1}(t)\right\}_{t \geq 0}$ and $\left\{Z_{2}(t)\right\}_{t \geq 0}$ are local martingales defined by

$$
\begin{aligned}
& Z_{1}(t)=\int_{0}^{t} \mathrm{e}^{-\left(\delta_{i}+q_{i}\right) s} \sigma_{i} m^{\prime}(Y(s), i) \mathrm{d} W(s), \\
& Z_{2}(t)=\int_{0}^{t} \mathrm{e}^{-\left(\delta_{i}+q_{i}\right) s}[m(Y(s-)-\pi(s), i)+\pi(s)-m(Y(s-), i)]\left(\mathrm{d} N_{i}(s)-\gamma_{i} \mathrm{~d} s\right) .
\end{aligned}
$$

However, the stopped processes $\left\{Z_{1}\left(t \wedge \tau_{a b}\right)\right\}_{t \geq 0}$ and $\left\{Z_{2}\left(t \wedge \tau_{a b}\right)\right\}_{t \geq 0}$ are martingales. Recall that $m(\cdot, i)$ is nonnegative. Taking conditional expectations of both sides of (4.3) yields

$$
m(x, i) \geq \mathrm{E}_{x}\left[\int_{0}^{t \wedge \tau_{a b}} \mathrm{e}^{-\left(\delta_{i}+q_{i}\right) s} \pi(s) \mathrm{d} N_{i}(s)+\int_{0}^{t \wedge \tau_{a b}} \mathrm{e}^{-\left(\delta_{i}+q_{i}\right) s} \sum_{j \neq i} q_{i j} u(Y(s), j) \mathrm{d} s\right] .
$$


Letting $a \rightarrow 0$ and $b \rightarrow \infty$, we obtain $\tau_{a} \rightarrow \tau_{i}$ and $\tau_{b} \rightarrow \infty$. Then $\tau_{a b} \rightarrow \tau_{i}$. Also, letting $t \rightarrow \infty$ and applying the dominated convergence theorem, we obtain

$$
\begin{aligned}
m(x, i) & \geq \mathrm{E}_{x}\left[\int_{0}^{\tau_{i}} \mathrm{e}^{-\left(\delta_{i}+q_{i}\right) s} \pi(s) \mathrm{d} N_{i}(s)+\int_{0}^{\tau_{i}} \mathrm{e}^{-\left(\delta_{i}+q_{i}\right) s} \sum_{j \neq i} q_{i j} u(Y(s), j) \mathrm{d} s\right] \\
& =M_{\pi}(x, i) .
\end{aligned}
$$

From the arbitrariness of strategy $\pi$ and the definition of $M(\cdot, i)$, we conclude that $m(x, i) \geq$ $M(x, i)$.

(ii) The proof of part (ii) is obvious from (i) and the definition of $M(\cdot, i)$.

\subsection{The modulated barrier strategy}

Motivated by [8] and [13], we consider the modulated barrier strategy. Let $\left\{T_{1}, T_{2}, \ldots\right\}$ be the times at which the dividends can be paid. Given the barrier level $\boldsymbol{b}=\left(b_{1}, b_{2}, \ldots, b_{K}\right)$, the modulated barrier strategy $\left\{\pi^{\boldsymbol{b}}(t)\right\}_{t \geq 0}$ is an $\mathcal{F}$-adapted process such that $\pi^{\boldsymbol{b}}\left(T_{i}\right)=\left(X\left(T_{i}\right)-\right.$ $\left.b_{J\left(T_{i}\right)}\right)^{+}$for $i=1,2, \ldots$.

To ease notation, let $M_{\boldsymbol{b}}(x, i)=M_{\pi^{b}}(x, i)$. We have the following propositions.

Proposition 4.1. Given $\boldsymbol{b}$, it holds that

$$
\begin{aligned}
M_{\boldsymbol{b}}(x, i)= & \gamma_{i} W_{i}^{\left(\theta_{i}\right)}(x)\left[\int_{0}^{b_{i}} M_{\boldsymbol{b}}(y, i) \mathrm{e}^{-r_{i} y} \mathrm{~d} y+\frac{\mathrm{e}^{-r_{i} b_{i}}}{r_{i}}\left(M_{\boldsymbol{b}}\left(b_{i}, i\right)+\frac{1}{r_{i}}\right)\right] \\
& -\gamma_{i} \int_{0}^{x} M_{\boldsymbol{b}}(y, i) W_{i}^{\left(\theta_{i}\right)}(x-y) \mathrm{d} y+W_{i}^{\left(\theta_{i}\right)}(x) \int_{0}^{\infty} \mathrm{e}^{-r_{i} y} \sum_{j \neq i} q_{i j} u(y, j) \mathrm{d} y \\
& -\int_{0}^{x} W_{i}^{\left(\theta_{i}\right)}(x-y) \sum_{j \neq i} q_{i j} u(y, j) \mathrm{d} y, \quad 0 \leq x<b_{i},
\end{aligned}
$$

and

$$
\begin{aligned}
M_{\boldsymbol{b}}(x, i)= & \gamma_{i} W_{i}^{\left(\theta_{i}\right)}(x)\left[\int_{0}^{b_{i}} M_{\boldsymbol{b}}(y, i) \mathrm{e}^{-r_{i} y} \mathrm{~d} y+\frac{\mathrm{e}^{-r_{i} b_{i}}}{r_{i}}\left(M_{\boldsymbol{b}}\left(b_{i}, i\right)+\frac{1}{r_{i}}\right)\right] \\
& -\gamma_{i}\left[\int_{0}^{b_{i}} M_{\boldsymbol{b}}(y, i) W_{i}^{\left(\theta_{i}\right)}(x-y) \mathrm{d} y+\int_{b_{i}}^{x}\left(y-b+M_{\boldsymbol{b}}\left(b_{i}, i\right)\right) W_{i}^{\left(\theta_{i}\right)}(x-y) \mathrm{d} y\right] \\
& +W_{i}^{\left(\theta_{i}\right)}(x) \int_{0}^{\infty} \mathrm{e}^{-r_{i} y} \sum_{j \neq i} q_{i j} u(y, j) \mathrm{d} y \\
& -\int_{0}^{x} W_{i}^{\left(\theta_{i}\right)}(x-y) \sum_{j \neq i} q_{i j} u(y, j) \mathrm{d} y, \quad x \geq b_{i},
\end{aligned}
$$

where $\theta_{i}=\delta_{i}+q_{i}+\gamma_{i}$

$$
W_{i}^{\left(\theta_{i}\right)}(x)=\frac{2}{\sigma_{i}^{2}} \frac{\mathrm{e}^{r_{i} x}-\mathrm{e}^{s_{i} x}}{r_{i}-s_{i}},
$$

and $r_{i}>0$ and $s_{i}<0$ are the solutions of the equation $\frac{1}{2} \sigma_{i}^{2} r^{2}+\mu_{i} r-\theta_{i}=0$.

Proof. Denote by $\left\{Y_{\boldsymbol{b}}(t)\right\}_{t \geq 0}$ and $\tau_{\boldsymbol{b}}$ process (3.4) and the time of ruin corresponding to the modulated barrier strategy $\boldsymbol{b}$, respectively. Let $Y^{\prime}(t)=x+X_{i}(t)$, and let $\tau$ be the time 
of ruin of $\left\{Y^{\prime}(t)\right\}_{t>0}$. Let $T_{1}$ be the first time at which the dividend is paid. Then $\left(Y^{\prime}(t)\right.$, $\left.t<\tau \wedge \eta\left(\gamma_{i}\right)\right)$ is in distribution equal to $\left(Y_{\boldsymbol{b}}(t), t<\tau_{\boldsymbol{b}} \wedge T_{1}\right)$. To simplify the notation, let $f(x, i)=\sum_{j \neq i} q_{i j} u(x, j)$ and $g(y)=y-\left(y-b_{i}\right)^{+}$. Noting that $M_{\boldsymbol{b}}(0, i)=0$, we have

$$
\begin{aligned}
M_{\boldsymbol{b}}(x, i)= & \mathrm{E}_{x}\left[\int_{0}^{\tau_{\boldsymbol{b}} \wedge T_{1}} \mathrm{e}^{-\left(\delta_{i}+q_{i}\right) s} f\left(Y_{\boldsymbol{b}}(s), i\right) \mathrm{d} s\right]+\mathrm{E}_{x}\left[\mathbf{1}_{\left\{T_{1} \leq \tau_{\boldsymbol{b}}\right\}} \mathrm{e}^{-\left(\delta_{i}+q_{i}\right) T_{1}}\left(Y_{\boldsymbol{b}}\left(T_{1}-\right)-b_{i}\right)^{+}\right] \\
& +\mathrm{E}_{x}\left[\mathbf{1}_{\left\{T_{1} \leq \tau_{\boldsymbol{b}}\right\}} \mathrm{e}^{-\left(\delta_{i}+q_{i}\right) T_{1}} M_{\boldsymbol{b}}\left(g\left(Y_{\boldsymbol{b}}\left(T_{1}-\right)\right), i\right)\right] \\
= & \mathrm{E}_{x}\left[\int_{0}^{\tau \wedge \eta\left(\gamma_{i}\right)} \mathrm{e}^{-\left(\delta_{i}+q_{i}\right) s} f\left(Y^{\prime}(s), i\right) \mathrm{d} s\right] \\
& +\mathrm{E}_{x}\left[\mathbf{1}_{\left\{\eta\left(\gamma_{i}\right) \leq \tau\right\}} \mathrm{e}^{-\left(\delta_{i}+q_{i}\right) \eta\left(\gamma_{i}\right)}\left(Y^{\prime}\left(\eta\left(\gamma_{i}\right)\right)-b_{i}\right)^{+}\right] \\
& +\mathrm{E}_{x}\left[\mathbf{1}_{\left\{\eta\left(\gamma_{i}\right) \leq \tau\right\}} \mathrm{e}^{-\left(\delta_{i}+q_{i}\right) \eta\left(\gamma_{i}\right)} M_{\boldsymbol{b}}\left(g\left(Y^{\prime}\left(\eta\left(\gamma_{i}\right)\right)\right), i\right)\right] \\
= & \mathrm{E}_{x}\left[\int_{0}^{\infty} \mathbf{1}_{\{s \leq \tau\}} \mathrm{e}^{-\theta_{i} s} f\left(Y^{\prime}(s), i\right) \mathrm{d} s\right]+\gamma_{i} \mathrm{E}_{x}\left[\int_{0}^{\infty} \mathbf{1}_{\{s \leq \tau\}} \mathrm{e}^{-\theta_{i} s}\left(Y^{\prime}(s)-b_{i}\right)^{+} \mathrm{d} s\right] \\
& +\gamma_{i} \mathrm{E}_{x}\left[\int_{0}^{\infty} \mathbf{1}_{\{s \leq \tau\}} \mathrm{e}^{-\theta_{i} s} M_{\boldsymbol{b}}\left(g\left(Y^{\prime}(s)\right), i\right) \mathrm{d} s\right] \\
= & \int_{0}^{\infty}\left[f(y, i)+\gamma_{i}\left(y-b_{i}\right)^{+}+\gamma_{i} M_{\boldsymbol{b}}(g(y), i)\right] \mathrm{d} y \\
& \times \int_{0}^{\infty} \mathrm{e}^{-\theta_{i} s} \mathrm{P}_{x}\left(Y^{\prime}(s) \in \mathrm{d} y, s<\tau\right) \mathrm{d} s .
\end{aligned}
$$

From Corollary 8.8 of [9] (or let $b \rightarrow \infty$ in Equation (4.4) of [8], noting that the left-hand side of their Equation (4.4) should be divided by $\theta_{i}$ ), we have

$$
\int_{0}^{\infty} \mathrm{e}^{-\theta_{i} s} \mathrm{P}_{x}\left(Y^{\prime}(s) \in \mathrm{d} y, s<\tau\right) \mathrm{d} s=\left[W_{i}^{\left(\theta_{i}\right)}(x) \mathrm{e}^{-r_{i} y}-\mathbf{1}_{\{x \geq y\}} W_{i}^{\left(\theta_{i}\right)}(x-y)\right] \mathrm{d} y .
$$

Inserting (4.7) into (4.6) yields (4.4) and (4.5).

Proposition 4.2. The function $M_{\boldsymbol{b}}(x, i) \in C^{2}([0, \infty))$ and satisfies

$$
\frac{\sigma_{i}^{2}}{2} M_{\boldsymbol{b}}^{\prime \prime}(x, i)+\mu_{i} M_{\boldsymbol{b}}^{\prime}(x, i)-\left(\delta_{i}+q_{i}\right) M_{\boldsymbol{b}}(x, i)+\sum_{j \neq i} q_{i j} u(x, j)=0
$$

for $0 \leq x<b_{i}$ and

$$
\frac{\sigma_{i}^{2}}{2} M_{\boldsymbol{b}}^{\prime \prime}(x, i)+\mu_{i} M_{\boldsymbol{b}}^{\prime}(x, i)-\theta_{i} M_{\boldsymbol{b}}(x, i)+\gamma_{i}\left[M_{\boldsymbol{b}}\left(b_{i}, i\right)+x-b_{i}\right]+\sum_{j \neq i} q_{i j} u(x, j)=0
$$

for $x \geq b_{i}$.

Proof. Noting that $W_{i}^{\left(\theta_{i}\right)}(x) \in C^{2}([0, \infty))$, we know that $M_{\boldsymbol{b}}(x, i) \in C^{2}\left(\left[0, b_{i}\right)\right)$ and $M_{\boldsymbol{b}}(x, i) \in C^{2}\left(\left[b_{i}, \infty\right)\right)$. Taking first- and second-order derivatives of (4.4) and (4.5), it is easy to check that $M_{\boldsymbol{b}}^{\prime \prime}(x, i)$ is continuous at $b_{i}$. Furthermore, using

$$
\frac{\sigma_{i}^{2}}{2} W_{i}^{\left(\theta_{i}\right)^{\prime \prime}}(x)+\mu_{i} W_{i}^{\left(\theta_{i}\right)^{\prime}}(x)-\theta_{i} W_{i}^{\left(\theta_{i}\right)}(x)=0,
$$

it is easy to show (4.8) and (4.9) (for simplicity, we omit the calculation details). 
From Proposition 4.2, if $\boldsymbol{u}(x) \in \mathbb{D}$ then we have $M_{\boldsymbol{b}}(x, i) \in C^{2}([0, \infty))$ for all $i \in \mathbb{J}$. Since later we will start with $\boldsymbol{U}_{0} \equiv \mathbf{0} \in \mathbb{D}$, we can work with $\boldsymbol{u} \in \mathbb{D} \cap C^{2}([0, \infty))$ in the following.

For $x \geq b_{i}$, it is easy to rewrite (4.5) as

$$
\begin{aligned}
M_{\boldsymbol{b}}(x, i)= & \frac{2 \gamma_{i} \mathrm{e}^{s_{i} x}}{\sigma_{i}^{2}\left(r_{i}-s_{i}\right)} \\
& \times\left[\int_{0}^{b_{i}} M_{\boldsymbol{b}}(y, i)\left(\mathrm{e}^{-s_{i} y}-\mathrm{e}^{-r_{i} y}\right) \mathrm{d} y+\left(\frac{1}{s_{i}} \mathrm{e}^{-s_{i} b_{i}}-\frac{1}{r_{i}} \mathrm{e}^{-r_{i} b_{i}}\right) M_{\boldsymbol{b}}\left(b_{i}, i\right)\right] \\
& +\frac{2 \gamma_{i} \mathrm{e}^{s_{i} x}}{\sigma_{i}^{2}\left(r_{i}-s_{i}\right)}\left(\frac{1}{s_{i}^{2}} \mathrm{e}^{-s_{i} b_{i}}-\frac{1}{r_{i}^{2}} \mathrm{e}^{-r_{i} b_{i}}\right)+a_{i}\left(x+M_{\boldsymbol{b}}\left(b_{i}, i\right)-b_{i}+\frac{\mu_{i}}{\theta_{i}}\right) \\
& +\Gamma_{i}(x),
\end{aligned}
$$

where $a_{i}=\gamma_{i} / \theta_{i}$ and

$$
\begin{aligned}
\Gamma_{i}(x)= & \frac{2 \mathrm{e}^{s_{i} x}}{\sigma_{i}^{2}\left(r_{i}-s_{i}\right)} \int_{0}^{x}\left(\mathrm{e}^{-s_{i} y}-\mathrm{e}^{-r_{i} y}\right) \sum_{j \neq i} q_{i j} u(y, j) \mathrm{d} y \\
& +\frac{2\left(\mathrm{e}^{r_{i} x}-\mathrm{e}^{s_{i} x}\right)}{\sigma_{i}^{2}\left(r_{i}-s_{i}\right)} \int_{x}^{\infty} \mathrm{e}^{-r_{i} y} \sum_{j \neq i} q_{i j} u(y, j) \mathrm{d} y .
\end{aligned}
$$

Corollary 4.1. For any $\boldsymbol{u} \in \mathbb{D}$, we have

(i) for any $\theta>0, \mathrm{e}^{-\theta x} M_{\boldsymbol{b}}(x, i) \rightarrow 0$ as $x \rightarrow \infty$;

(ii) $M_{\boldsymbol{b}}^{\prime}(x, i) \rightarrow a_{i}+\left(1 / \theta_{i}\right) \sum_{j \neq i} q_{i j} u^{\prime}(\infty, j)$ and $M_{\boldsymbol{b}}^{\prime \prime}(x, i) \rightarrow 0$ as $x \rightarrow \infty$.

Proof. (i) Since $\Gamma_{i}(x) \geq 0$, it follows from (4.10) that $M_{\boldsymbol{b}}(x, i) \rightarrow \infty$ as $x \rightarrow \infty$. Recall that if $\boldsymbol{u} \in \mathbb{D}$ then, for any $\theta>0$ and $i \in \mathbb{J}, \mathrm{e}^{-\theta x} u(x, i) \rightarrow 0$ as $x \rightarrow \infty$. It holds that

$$
\begin{aligned}
\Gamma_{i}(x)= & \frac{2 \mathrm{e}^{s_{i} x}}{\sigma_{i}^{2}\left(r_{i}-s_{i}\right) s_{i}} \int_{0}^{x} \mathrm{e}^{-s_{i} y} \sum_{j \neq i} q_{i j} u^{\prime}(y, j) \mathrm{d} y \\
& +\frac{2 \mathrm{e}^{r_{i} x}}{\sigma_{i}^{2}\left(r_{i}-s_{i}\right) r_{i}} \int_{x}^{\infty} \mathrm{e}^{-r_{i} y} \sum_{j \neq i} q_{i j} u^{\prime}(y, j) \mathrm{d} y \\
& -\frac{2 \mathrm{e}^{s_{i} x}}{\sigma_{i}^{2}\left(r_{i}-s_{i}\right) r_{i}} \int_{0}^{\infty} \mathrm{e}^{-r_{i} y} \sum_{j \neq i} q_{i j} u^{\prime}(y, j) \mathrm{d} y+\frac{1}{\theta_{i}} \sum_{j \neq i} q_{i j} u(x, j) .
\end{aligned}
$$

Note that

$$
\int_{0}^{x} \mathrm{e}^{-s_{i} y} \sum_{j \neq i} q_{i j} u^{\prime}(y, j) \mathrm{d} y \rightarrow \infty \quad \text { and } \quad \int_{x}^{\infty} \mathrm{e}^{-r_{i} y} \sum_{j \neq i} q_{i j} u^{\prime}(y, j) \mathrm{d} y \rightarrow 0 \quad \text { as } x \rightarrow \infty .
$$

Hence, by l'Hôpital's rule,

$$
\Gamma_{i}(x) \rightarrow \frac{\mu_{i}}{\theta_{i}^{2}} \sum_{j \neq i} q_{i j} u^{\prime}(\infty, j)+\frac{1}{\theta_{i}} \sum_{j \neq i} q_{i j} u(\infty, j) \quad \text { as } x \rightarrow \infty .
$$

Thus, by (4.10), for any $\theta>0, \mathrm{e}^{-\theta x} M_{\boldsymbol{b}}(x, i) \rightarrow 0$ as $x \rightarrow \infty$. 
(ii) Similarly, for any $\boldsymbol{u} \in \mathbb{D}$, by l'Hôpital's rule,

$$
\begin{aligned}
\Gamma_{i}^{\prime}(x)= & \frac{2 \mathrm{e}^{s_{i} x}}{\sigma_{i}^{2}\left(r_{i}-s_{i}\right)} \int_{0}^{x} \mathrm{e}^{-s_{i} y} \sum_{j \neq i} q_{i j} u^{\prime}(y, j) \mathrm{d} y \\
& +\frac{2 \mathrm{e}^{r_{i} x}}{\sigma_{i}^{2}\left(r_{i}-s_{i}\right)} \int_{x}^{\infty} \mathrm{e}^{-r_{i} y} \sum_{j \neq i} q_{i j} u^{\prime}(y, j) \mathrm{d} y \\
& -\frac{2 s_{i} \mathrm{e}^{s_{i} x}}{\sigma_{i}^{2}\left(r_{i}-s_{i}\right) r_{i}} \int_{0}^{\infty} \mathrm{e}^{-r_{i} y} \sum_{j \neq i} q_{i j} u^{\prime}(y, j) \mathrm{d} y \\
\rightarrow & \frac{1}{\theta_{i}} \sum_{j \neq i} q_{i j} u^{\prime}(\infty, j) \quad \text { as } x \rightarrow \infty .
\end{aligned}
$$

Thus, by (4.10), it is easy to see that $M_{b}^{\prime}(x, i) \rightarrow a_{i}+\left(1 / \theta_{i}\right) \sum_{j \neq i} q_{i j} u^{\prime}(\infty, j)$ as $x \rightarrow \infty$.

Also,

$$
\begin{aligned}
\Gamma_{i}^{\prime \prime}(x)= & \frac{2 s_{i} \mathrm{e}^{s_{i} x}}{\sigma_{i}^{2}\left(r_{i}-s_{i}\right)} \int_{0}^{x} \mathrm{e}^{-s_{i} y} \sum_{j \neq i} q_{i j} u^{\prime}(y, j) \mathrm{d} y \\
& +\frac{2 r_{i} \mathrm{e}^{r_{i} x}}{\sigma_{i}^{2}\left(r_{i}-s_{i}\right)} \int_{x}^{\infty} \mathrm{e}^{-r_{i} y} \sum_{j \neq i} q_{i j} u^{\prime}(y, j) \mathrm{d} y \\
& -\frac{2 s_{i}^{2} \mathrm{e}^{s_{i} x}}{\sigma_{i}^{2}\left(r_{i}-s_{i}\right) r_{i}} \int_{0}^{\infty} \mathrm{e}^{-r_{i} y} \sum_{j \neq i} q_{i j} u^{\prime}(y, j) \mathrm{d} y \\
\rightarrow & 0 \text { as } x \rightarrow \infty .
\end{aligned}
$$

From Section 2.1.1 of [10], the solution of (4.8) is given by

$$
M_{\boldsymbol{b}}(x, i)=A_{i} \mathrm{e}^{\alpha_{i} x}+B_{i} \mathrm{e}^{\beta_{i} x}-\int_{0}^{x} \frac{2\left(\mathrm{e}^{\alpha_{i}(x-y)}-\mathrm{e}^{\beta_{i}(x-y)}\right)}{\sigma_{i}^{2}\left(\alpha_{i}-\beta_{i}\right)} \sum_{j \neq i} q_{i j} u(y, j) \mathrm{d} y,
$$

where $A_{i}$ and $B_{i}$ are constants to be determined, and $\alpha_{i}>0$ and $\beta_{i}<0$ are the solutions of the equation

$$
\frac{1}{2} \sigma_{i}^{2} r^{2}+\mu_{i} r-\left(\delta_{i}+q_{i}\right)=0
$$

The solution of (4.9) is given by

$$
\begin{aligned}
M_{\boldsymbol{b}}(x, i)= & C_{i} \mathrm{e}^{r_{i}\left(x-b_{i}\right)}+D_{i} \mathrm{e}^{s_{i}\left(x-b_{i}\right)}-\int_{b_{i}}^{x} \frac{2\left(\mathrm{e}^{r_{i}(x-y)}-\mathrm{e}^{s_{i}(x-y)}\right)}{\sigma_{i}^{2}\left(r_{i}-s_{i}\right)} \sum_{j \neq i} q_{i j} u(y, j) \mathrm{d} y \\
& +a_{i} x+c_{i}
\end{aligned}
$$

where $C_{i}$ and $D_{i}$ are constants to be determined, and

$$
c_{i}=\frac{\mu_{i} a_{i}+\gamma_{i}\left[m\left(b_{i}-, i\right)-b_{i}\right]}{\delta_{i}+q_{i}+\gamma_{i}} .
$$


From (4.12) we have

$$
\begin{aligned}
M_{\boldsymbol{b}}^{\prime \prime}(x, i)= & C_{i} r_{i}^{2} \mathrm{e}^{r_{i}\left(x-b_{i}\right)}+D_{i} s_{i}^{2} \mathrm{e}^{s_{i}\left(x-b_{i}\right)}-\frac{2}{\sigma_{i}^{2}} \sum_{j \neq i} q_{i j} u(x, j) \\
& -\int_{b_{i}}^{x} \frac{2\left(r_{i}^{2} \mathrm{e}^{r_{i}(x-y)}-s_{i}^{2} \mathrm{e}^{s_{i}(x-y)}\right)}{\sigma_{i}^{2}\left(r_{i}-s_{i}\right)} \sum_{j \neq i} q_{i j} u(y, j) \mathrm{d} y \\
= & {\left[C_{i} r_{i}^{2}-\frac{2 r_{i}}{\sigma_{i}^{2}\left(r_{i}-s_{i}\right)} \Xi_{i}\left(b_{i}\right)\right] \mathrm{e}^{r_{i}\left(x-b_{i}\right)} } \\
& +\left[D_{i} s_{i}^{2}+\frac{2 s_{i}}{\sigma_{i}^{2}\left(r_{i}-s_{i}\right)} \sum_{j \neq i} q_{i j} u\left(b_{i}, j\right)\right] \mathrm{e}^{s_{i}\left(x-b_{i}\right)} \\
& +\frac{2}{\sigma_{i}^{2}\left(r_{i}-s_{i}\right)}\left[s_{i} \mathrm{e}^{s_{i} x} \int_{0}^{x} \mathrm{e}^{-s_{i} y} \sum_{j \neq i} q_{i j} u^{\prime}(y, j) \mathrm{d} y\right. \\
& \left.+r_{i} \mathrm{e}^{r_{i} x} \int_{x}^{\infty} \mathrm{e}^{-r_{i} y} \sum_{j \neq i} q_{i j} u^{\prime}(y, j) \mathrm{d} y\right],
\end{aligned}
$$

where

$$
\Xi_{i}\left(b_{i}\right)=\sum_{j \neq i} q_{i j} u\left(b_{i}, j\right)+\int_{b_{i}}^{\infty} \mathrm{e}^{r_{i}\left(b_{i}-y\right)} \sum_{j \neq i} q_{i j} u^{\prime}(y, j) \mathrm{d} y .
$$

Since

$$
s_{i} \mathrm{e}^{s_{i} x} \int_{0}^{x} \mathrm{e}^{-s_{i} y} \sum_{j \neq i} q_{i j} u^{\prime}(y, j) \mathrm{d} y+r_{i} \mathrm{e}^{r_{i} x} \int_{x}^{\infty} \mathrm{e}^{-r_{i} y} \sum_{j \neq i} q_{i j} u^{\prime}(y, j) \mathrm{d} y \rightarrow 0 \quad \text { as } x \rightarrow \infty,
$$

it follows from Corollary 4.1(ii) that

$$
C_{i}=\frac{2}{\sigma_{i}^{2}\left(r_{i}-s_{i}\right) r_{i}} \Xi_{i}\left(b_{i}\right) .
$$

Since $M_{\boldsymbol{b}}(0, i)=0$, from (4.11) we know that $B_{i}=-A_{i}$. From the smooth-fit conditions,

$$
M_{\boldsymbol{b}}\left(b_{i}-, i\right)=M_{\boldsymbol{b}}\left(b_{i}+, i\right) \quad \text { and } \quad M_{\boldsymbol{b}}^{\prime}\left(b_{i}-, i\right)=M_{\boldsymbol{b}}^{\prime}\left(b_{i}+, i\right),
$$

we have

$$
\begin{aligned}
A_{i} & =\left[s_{i} \frac{\delta_{i}+q_{i}}{\theta_{i}} h_{i}\left(b_{i}\right)-h_{i}^{\prime}\left(b_{i}\right)\right]^{-1}\left[\Lambda_{i}\left(b_{i}\right)-\frac{2}{\sigma_{i}^{2} r_{i}} \Xi_{i}\left(b_{i}\right)+s_{i} \frac{\mu_{i} a_{i}}{\theta_{i}}-a_{i}\right], \\
D_{i} & =\frac{\delta_{i}+q_{i}}{\theta_{i}}\left[A_{i} h_{i}\left(b_{i}\right)-\int_{0}^{b_{i}} W_{i}^{\left(\delta_{i}+q_{i}\right)}\left(b_{i}-y\right) \sum_{j \neq i} q_{i j} u(y, j) \mathrm{d} y\right]-C_{i}-\frac{\mu_{i} a_{i}}{\theta_{i}},
\end{aligned}
$$

where $h_{i}\left(b_{i}\right)=\mathrm{e}^{\alpha_{i} b_{i}}-\mathrm{e}^{\beta_{i} b_{i}}$ and

$$
\begin{aligned}
\Lambda_{i}\left(b_{i}\right)= & s_{i} \frac{\delta_{i}+q_{i}}{\theta_{i}} \int_{0}^{b_{i}} W_{i}^{\left(\delta_{i}+q_{i}\right)}\left(b_{i}-y\right) \sum_{j \neq i} q_{i j} u(y, j) \mathrm{d} y \\
& -\int_{0}^{b_{i}} W_{i}^{\left(\delta_{i}+q_{i}\right)^{\prime}}\left(b_{i}-y\right) \sum_{j \neq i} q_{i j} u(y, j) \mathrm{d} y .
\end{aligned}
$$


Now we consider the optimal modulated barrier strategy, i.e. we want to find the $b_{i}$ that maximizes $A_{i}$. For convenience, we define the function

$$
A_{i}(b)=\left[s_{i} \frac{\delta_{i}+q_{i}}{\theta_{i}} h_{i}(b)-h_{i}^{\prime}(b)\right]^{-1} \Delta_{i}(b),
$$

where

$$
\Delta_{i}(b)=\Lambda_{i}(b)-\frac{2}{\sigma_{i}^{2} r_{i}} \Xi_{i}(b)+s_{i} \frac{\mu_{i} a_{i}}{\theta_{i}}-a_{i} .
$$

Then the first-order condition $A_{i}^{\prime}(b)=0$ implies that

$$
\Delta_{i}^{\prime}(b)\left[s_{i} \frac{\delta_{i}+q_{i}}{\theta_{i}} h_{i}(b)-h_{i}^{\prime}(b)\right]=\Delta_{i}(b)\left[s_{i} \frac{\delta_{i}+q_{i}}{\theta_{i}} h_{i}^{\prime}(b)-h_{i}^{\prime \prime}(b)\right] .
$$

In Appendix A we show that (4.13) admits a root in $(0, \infty)$. Note that, for any $x \geq 0$,

$$
\frac{\sigma_{i}^{2}}{2} h_{i}^{\prime \prime}(x)+\mu_{i} h_{i}^{\prime}(x)-\left(\delta_{i}+q_{i}\right) h_{i}(x)=0 .
$$

It follows from (4.13) and (4.14) that

$$
\left[\left(\delta_{i}+q_{i}\right)+\frac{\sigma_{i}^{2}}{2} s_{i} \frac{\delta_{i}+q_{i}}{\theta_{i}} \frac{\Delta_{i}^{\prime}(b)}{\Delta_{i}(b)}\right] h_{i}(b)=\left[\frac{\sigma_{i}^{2}}{2} s_{i} \frac{\delta_{i}+q_{i}}{\theta_{i}}+\mu_{i}+\frac{\sigma_{i}^{2}}{2} \frac{\Delta_{i}^{\prime}(b)}{\Delta_{i}(b)}\right] h_{i}^{\prime}(b) .
$$

Proposition 4.3. Let $b_{i}^{*}>0$ be a solution of (4.13). Then $M_{b^{*}}^{\prime}\left(b_{i}^{*}, i\right)=1$ and $M_{b^{*}}^{\prime \prime}\left(b_{i}^{*}, i\right) \leq 0$, where the ith element of $\boldsymbol{b}^{*}$ is $b_{i}^{*}$.

Proof. From (4.11) we know that

$$
\begin{aligned}
M_{\boldsymbol{b}^{*}}^{\prime}\left(b_{i}^{*}, i\right)= & A_{i}\left(b_{i}^{*}\right) h_{i}^{\prime}\left(b_{i}^{*}\right)-\int_{0}^{b_{i}^{*}} W_{i}^{\left(\delta_{i}+q_{i}\right)^{\prime}}\left(b_{i}^{*}-y\right) \sum_{j \neq i} q_{i j} u(y, j) \mathrm{d} y \\
= & h_{i}^{\prime}\left(b_{i}^{*}\right)\left[s_{i} \frac{\delta_{i}+q_{i}}{\theta_{i}} h_{i}\left(b_{i}^{*}\right)-h_{i}^{\prime}\left(b_{i}^{*}\right)\right]^{-1} \Delta_{i}\left(b_{i}^{*}\right) \\
& -\int_{0}^{b_{i}^{*}} W_{i}^{\left(\delta_{i}+q_{i}\right)^{\prime}}\left(b_{i}^{*}-y\right) \sum_{j \neq i} q_{i j} u(y, j) \mathrm{d} y .
\end{aligned}
$$

It follows from (4.15) that

$$
h_{i}^{\prime}\left(b_{i}^{*}\right)\left[s_{i} \frac{\delta_{i}+q_{i}}{\theta_{i}} h_{i}\left(b_{i}^{*}\right)-h_{i}^{\prime}\left(b_{i}^{*}\right)\right]^{-1}=\frac{\theta_{i} \Delta_{i}\left(b_{i}^{*}\right)+\sigma_{i}^{2} s_{i} \Delta_{i}^{\prime}\left(b_{i}^{*}\right) / 2}{\Delta_{i}\left(b_{i}^{*}\right)\left(s_{i} \mu_{i}-\theta_{i}\right) a_{i}} .
$$

The above equation yields

$$
\begin{aligned}
M_{\boldsymbol{b}^{*}}^{\prime}\left(b_{i}^{*}, i\right)= & \frac{\theta_{i} \Delta_{i}\left(b_{i}^{*}\right)+\sigma_{i}^{2} s_{i} \Delta_{i}^{\prime}\left(b_{i}^{*}\right) / 2}{\left(s_{i} \mu_{i}-\theta_{i}\right) a_{i}}-\int_{0}^{b_{i}^{*}} W_{i}^{\left(\delta_{i}+q_{i}\right)^{\prime}}\left(b_{i}^{*}-y\right) \sum_{j \neq i} q_{i j} u(y, j) \mathrm{d} y \\
= & 1-\frac{2 \theta_{i}}{\sigma_{i}^{2} s_{i}^{2} a_{i}}\left[\Lambda_{i}\left(b_{i}^{*}\right)-\frac{2}{\sigma_{i}^{2} r_{i}} \Xi_{i}\left(b_{i}^{*}\right)\right]-\frac{1}{s_{i} a_{i}}\left[\Lambda_{i}^{\prime}\left(b_{i}^{*}\right)-\frac{2}{\sigma_{i}^{2} r_{i}} \Xi_{i}^{\prime}\left(b_{i}^{*}\right)\right] \\
& -\int_{0}^{b_{i}^{*}} W_{i}^{\left(\delta_{i}+q_{i}\right)^{\prime}}\left(b_{i}^{*}-y\right) \sum_{j \neq i} q_{i j} u(y, j) \mathrm{d} y \\
= & 1,
\end{aligned}
$$


where the last equality follows from

$$
\frac{\sigma_{i}^{2}}{2} W_{i}^{\left(\delta_{i}+q_{i}\right)^{\prime \prime}}(x)+\mu_{i} W_{i}^{\left(\delta_{i}+q_{i}\right)^{\prime}}(x)-\left(\delta_{i}+q_{i}\right) W_{i}^{\left(\delta_{i}+q_{i}\right)}(x)=0 \quad \text { for } x \geq 0 .
$$

Thus, it is easy to see that

$$
A_{i}\left(b_{i}^{*}\right)=\frac{1}{h_{i}^{\prime}\left(b_{i}^{*}\right)}\left[1+\int_{0}^{b_{i}^{*}} W_{i}^{\left(\delta_{i}+q_{i}\right)^{\prime}}\left(b_{i}^{*}-y\right) \sum_{j \neq i} q_{i j} u(y, j) \mathrm{d} y\right] .
$$

Consequently, from (4.11) we have

$$
\begin{aligned}
M_{\boldsymbol{b}^{*}}^{\prime \prime}\left(b_{i}^{*}, i\right)= & A_{i}\left(b_{i}^{*}\right) h_{i}^{\prime \prime}\left(b_{i}^{*}\right)-\int_{0}^{b_{i}^{*}} W_{i}^{\left(\delta_{i}+q_{i}\right)^{\prime \prime}}\left(b_{i}^{*}-y\right) \sum_{j \neq i} q_{i j} u(y, j) \mathrm{d} y \\
& -\frac{2}{\sigma_{i}^{2}} \sum_{j \neq i} q_{i j} u\left(b_{i}^{*}, j\right) \\
= & \frac{h_{i}^{\prime \prime}\left(b_{i}^{*}\right)}{h_{i}^{\prime}\left(b_{i}^{*}\right)}\left[1+\int_{0}^{b_{i}^{*}} W_{i}^{\left(\delta_{i}+q_{i}\right)^{\prime}}\left(b_{i}^{*}-y\right) \sum_{j \neq i} q_{i j} u(y, j) \mathrm{d} y\right] \\
& -\int_{0}^{b_{i}^{*}} W_{i}^{\left(\delta_{i}+q_{i}\right)^{\prime}}\left(b_{i}^{*}-y\right) \sum_{j \neq i} q_{i j} u^{\prime}(y, j) \mathrm{d} y
\end{aligned}
$$

Noting that $h_{i}^{\prime}\left(b_{i}^{*}\right) \geq 0$,

$$
\int_{0}^{b_{i}^{*}} W_{i}^{\left(\delta_{i}+q_{i}\right)^{\prime}}\left(b_{i}^{*}-y\right) \sum_{j \neq i} q_{i j} u(y, j) \mathrm{d} y \geq 0,
$$

and

$$
\int_{0}^{b_{i}^{*}} W_{i}^{\left(\delta_{i}+q_{i}\right)^{\prime}}\left(b_{i}^{*}-y\right) \sum_{j \neq i} q_{i j} u^{\prime}(y, j) \mathrm{d} y \geq 0,
$$

it is sufficient to show that $h_{i}^{\prime \prime}\left(b_{i}^{*}\right) \leq 0$. From (4.14) and (4.15), we have

$$
\begin{aligned}
h_{i}^{\prime \prime}\left(b_{i}^{*}\right) & =h_{i}^{\prime}\left(b_{i}^{*}\right)\left\{\left[s_{i} \frac{\delta_{i}+q_{i}}{\theta_{i}}+\frac{2}{\sigma_{i}^{2}} \mu_{i}+\frac{\Delta_{i}^{\prime}\left(b_{i}^{*}\right)}{\Delta_{i}\left(b_{i}^{*}\right)}\right]\left[1+\frac{\sigma_{i}^{2}}{2} \frac{s_{i}}{\theta_{i}} \frac{\Delta_{i}^{\prime}\left(b_{i}^{*}\right)}{\Delta_{i}\left(b_{i}^{*}\right)}\right]^{-1}-\frac{2}{\sigma_{i}^{2}} \mu_{i}\right\} \\
& =h_{i}^{\prime}\left(b_{i}^{*}\right)\left[s_{i} \frac{\delta_{i}+q_{i}}{\theta_{i}} \Delta_{i}\left(b_{i}^{*}\right)-\frac{\sigma_{i}^{2}}{2} \frac{s_{i}^{2}}{\theta_{i}} \Delta_{i}^{\prime}\left(b_{i}^{*}\right)\right]\left[\Delta_{i}\left(b_{i}^{*}\right)+\frac{\sigma_{i}^{2}}{2} \frac{s_{i}}{\theta_{i}} \Delta_{i}^{\prime}\left(b_{i}^{*}\right)\right]^{-1} .
\end{aligned}
$$

Noting that $\Delta_{i}\left(b_{i}^{*}\right) \leq 0, \Delta_{i}^{\prime}\left(b_{i}^{*}\right) \leq 0$, and

$$
\begin{aligned}
\Delta_{i}\left(b_{i}^{*}\right)+\frac{\sigma_{i}^{2}}{2} \frac{s_{i}}{\theta_{i}} \Delta_{i}^{\prime}\left(b_{i}^{*}\right)= & s_{i} \frac{\delta_{i}+q_{i}}{\theta_{i}} \int_{0}^{b_{i}^{*}} W_{i}^{\left(\delta_{i}+q_{i}\right)}\left(b_{i}^{*}-y\right) \sum_{j \neq i} q_{i j} u(y, j) \mathrm{d} y \\
& -\int_{0}^{b_{i}^{*}} W_{i}^{\left(\delta_{i}+q_{i}\right)^{\prime}}\left(b_{i}^{*}-y\right) \sum_{j \neq i} q_{i j} u(y, j) \mathrm{d} y \\
& +\frac{\sigma_{i}^{2}}{2} s_{i}^{2} \frac{\delta_{i}+q_{i}}{\theta_{i}^{2}} \int_{0}^{b_{i}^{*}} W_{i}^{\left(\delta_{i}+q_{i}\right)^{\prime}}\left(b_{i}^{*}-y\right) \sum_{j \neq i} q_{i j} u(y, j) \mathrm{d} y
\end{aligned}
$$




$$
\begin{aligned}
& -\frac{\sigma_{i}^{2}}{2} \frac{s_{i}}{\theta_{i}} \int_{0}^{b_{i}^{*}} W_{i}^{\left(\delta_{i}+q_{i}\right)^{\prime \prime}}\left(b_{i}^{*}-y\right) \sum_{j \neq i} q_{i j} u(y, j) \mathrm{d} y \\
& -\left(\frac{2}{\sigma_{i}^{2} r_{i}}+\frac{s_{i}}{\theta_{i}}\right) \Xi_{i}\left(b_{i}^{*}\right)+\frac{a_{i}}{\theta_{i}}\left(\mu_{i} s_{i}-\theta_{i}\right) \\
= & -\frac{a_{i}}{\theta_{i}} \frac{\sigma_{i}^{2}}{2} s_{i}^{2}\left[\int_{0}^{b_{i}^{*}} W_{i}^{\left(\delta_{i}+q_{i}\right)^{\prime}}\left(b_{i}^{*}-y\right) \sum_{j \neq i} q_{i j} u(y, j) \mathrm{d} y+1\right] \\
< & 0,
\end{aligned}
$$

where the second equality follows from (4.16) and $\mu_{i} s_{i}-\theta_{i}=-\sigma_{i}^{2} s_{i}^{2} / 2$, we have $h_{i}^{\prime \prime}\left(b_{i}^{*}\right) \leq 0$, which completes the proof.

Proposition 4.4. The function $M_{\boldsymbol{b}^{*}}(x, i)$ is increasing and concave on $[0, \infty)$.

Proof. Define

$$
\xi(x, i)=M_{b^{*}}^{\prime \prime}(x, i)
$$

Note that $\xi(x, i) \in C^{1}([0, \infty)), \xi(x, i) \in C^{2}\left([0, \infty) \backslash\left\{b_{i}^{*}\right\}\right)$, and satisfies

$$
\begin{aligned}
& \frac{\sigma_{i}^{2}}{2} \xi^{\prime \prime}(x, i)+\mu_{i} \xi^{\prime}(x, i)-\left(\delta_{i}+q_{i}\right) \xi(x, i)+\sum_{j \neq i} q_{i j} u^{\prime \prime}(x, j)=0, \quad 0 \leq x \leq b_{i}^{*}, \\
& \frac{\sigma_{i}^{2}}{2} \xi^{\prime \prime}(x, i)+\mu_{i} \xi^{\prime}(x, i)-\left(\delta_{i}+q_{i}+\gamma_{i}\right) \xi(x, i)+\sum_{j \neq i} q_{i j} u^{\prime \prime}(x, j)=0, \quad x \geq b_{i}^{*} .
\end{aligned}
$$

Recall that $Y^{\prime}(t)=x+X_{i}(t)$. If $Y^{\prime}(0)=x \in\left(0, b_{i}^{*}\right)$ then define $\tau_{0, b_{i}^{*}}:=\inf \{t \geq 0$ : $\left.Y^{\prime}(t) \notin\left(0, b_{i}^{*}\right)\right\}$. From (4.8), we know that $M_{\boldsymbol{b}^{*}}^{\prime \prime}(0, i) \leq 0$. Thus, from Proposition 4.3 we have $\xi\left(Y^{\prime}\left(\tau_{0, b_{i}^{*}}\right), i\right) \leq 0$. Applying Itô's formula to $\mathrm{e}^{-\left(\delta_{i}+q_{i}\right) t} \xi\left(Y^{\prime}(t), i\right)$ yields

$$
\xi(x, i)=\mathrm{E}_{x}\left[\mathrm{e}^{-\left(\delta_{i}+q_{i}\right) \tau_{0, b_{i}^{*}}} \xi\left(Y^{\prime}\left(\tau_{0, b_{i}^{*}}\right), i\right)+\int_{0}^{\tau_{0, b_{i}^{*}}} \sum_{j \neq i} q_{i j} u^{\prime \prime}\left(Y^{\prime}(s), j\right) \mathrm{d} y\right] \leq 0 .
$$

If $Y^{\prime}(0)=x \in\left(b_{i}^{*}, \infty\right)$, define $\tau_{b_{i}^{*}}:=\inf \left\{t \geq 0: Y^{\prime}(t) \notin\left(b_{i}^{*}, \infty\right)\right\}$. Since $\mu_{i}>0$, we know that $Y^{\prime}(\infty)=\infty$. From Corollary 4.1(ii) and Proposition 4.3, we have $\xi\left(Y^{\prime}\left(\tau_{b_{i}^{*}}\right), i\right) \leq 0$. Similarly, applying Itô's formula to $\mathrm{e}^{-\left(\delta_{i}+q_{i}+\gamma_{i}\right) t} \xi\left(Y^{\prime}(t), i\right)$ yields $\xi(x, i) \leq 0$. Hence, we have proved the concavity of $M_{\boldsymbol{b}^{*}}(x, i)$.

It follows from Corollary 4.1(ii) that $M_{\boldsymbol{b}^{*}}^{\prime}(\infty, i)>0$. Therefore, the concavity of $M_{\boldsymbol{b}^{*}}(x, i)$ implies that $M_{\boldsymbol{b}^{*}}^{\prime}(x, i)>0$ for all $x \geq 0$, i.e. $M_{\boldsymbol{b}^{*}}(x, i)$ is increasing on $[0, \infty)$.

\subsection{Verification of $M_{b^{*}}(x, i)$}

In this subsection we verify that the modulated barrier strategy $\pi^{b^{*}}$ is optimal for the auxiliary problem (4.1).

From Proposition 4.1, it is easy to see that $M_{\boldsymbol{b}^{*}}(0, i)=0$. It follows from Propositions 4.2 and 4.4 that $M_{\boldsymbol{b}^{*}}(x, i) \in C^{2}([0, \infty))$ and is nonnegative.

From Proposition 4.3 and the concavity of $M_{b^{*}}(x, i)$ (see Proposition 4.4), it is easy to see that $M_{\boldsymbol{b}^{*}}^{\prime}(x, i) \geq 1$ for $x \in\left[0, b_{i}^{*}\right)$ and $M_{\boldsymbol{b}^{*}}^{\prime}(x, i) \leq 1$ for $x \in\left[b_{i}^{*}, \infty\right)$. Thus, the maximum

$$
\max _{0 \leq y \leq x}\left\{M_{\boldsymbol{b}^{*}}(x-y, i)+y\right\}
$$


is attained at $y=0$ if $x \in\left[0, b_{i}^{*}\right)$ and at $y=x-b_{i}^{*}$ if $x \in\left[b_{i}^{*}, \infty\right)$. Now, it follows from Proposition 4.2 that $M_{b^{*}}(x, i)$ satisfies the HJB equation (4.2).

We have shown that $M_{\boldsymbol{b}^{*}}(x, i)$ satisfies the conditions of Theorem 4.1. Therefore, $M_{\boldsymbol{b}^{*}}(x, i)$ is the value function of the auxiliary optimal problem (4.1), and the modulated barrier strategy $\pi^{b^{*}}$ is the optimal strategy.

We can now answer the question raised at the beginning of this section, i.e. what $\boldsymbol{U}_{n+1}$ is when $\boldsymbol{U}_{n}$ is given. From Corollary 4.1 and Proposition 4.4, we know that if $\boldsymbol{u}(x) \in \mathbb{D}$ then $\boldsymbol{M}_{\boldsymbol{b}^{*}}(x) \in \mathbb{D}$, where $\boldsymbol{M}_{\boldsymbol{b}^{*}}(x)=\left(M_{\boldsymbol{b}^{*}}(x, 1), M_{\boldsymbol{b}^{*}}(x, 2), \ldots, M_{\boldsymbol{b}^{*}}(x, K)\right)$ and $\boldsymbol{b}^{*}=$ $\left(b_{1}^{*}, b_{2}^{*}, \ldots, b_{K}^{*}\right)$. Obviously, $\mathbf{0} \in \mathbb{D}$. Thus, from the definition of $U_{n}(x, i)$, it is easy to see that $\boldsymbol{U}_{n}(x) \in \mathbb{D}$ for $n=0,1,2, \ldots$ Furthermore, when $\boldsymbol{U}_{n}(x)$ is given, $U_{n+1}(x, i)$ is given by (4.11) and (4.12) with $u$ replaced by $U_{n}$.

\section{Back to the original problem}

\subsection{The general cases}

We now consider the original problem (2.1). Since $\boldsymbol{U}_{n}(x) \in \mathbb{D}$ for $n=0,1,2, \ldots$, we know that $\boldsymbol{V}(x) \in \mathbb{D}$ as it is the pointwise limit of $\boldsymbol{U}_{n}(x)$. From the results given in Section 4 we know that a modulated barrier strategy $\pi^{\boldsymbol{b}}$ at some barrier level $\boldsymbol{b}=\left(b_{1}, b_{2}, \ldots, b_{K}\right)$ will be a maximizer of the right-hand side of (3.3). Recalling Remark 3.2, such a modulated barrier strategy is also the optimal strategy of the original problem (2.1).

There are two ways to obtain the value function and the optimal barrier levels. The first method is to implement the following iteration.

Step 1. Set $\boldsymbol{U}_{0}(x) \equiv 0$.

Step 2. Find $\boldsymbol{b}_{n+1}$ by (4.13), and find $\boldsymbol{U}_{n+1}(x)$ by (4.11) and (4.12).

Step 3. Stop when $\sup _{x \geq 0, i \in \mathbb{J}}\left|U_{n+1}(x, i)-U_{n}(x, i)\right|<\varepsilon$; otherwise, return to step 2, where $\varepsilon>0$ is the desirable level of accuracy.

The second method is to solve the system of differential equations. From (4.8) and (4.9), the value function $\boldsymbol{V}(x)$ and the optimal barrier levels $\boldsymbol{b}=\left(b_{1}, b_{2}, \ldots, b_{K}\right)$ satisfy

$$
\begin{aligned}
& \frac{\sigma_{i}^{2}}{2} V^{\prime \prime}(x, i)+\mu_{i} V^{\prime}(x, i)-\left(\delta_{i}+q_{i}\right) V(x, i)+\sum_{j \neq i} q_{i j} V(x, j) \\
& \quad=0 \quad \text { for } 0 \leq x<b_{i}, \\
& \frac{\sigma_{i}^{2}}{2} V^{\prime \prime}(x, i)+\mu_{i} V^{\prime}(x, i)-\theta_{i} V(x, i)+\gamma_{i}\left[V\left(b_{i}, i\right)+x-b_{i}\right]+\sum_{j \neq i} q_{i j} V(x, j) \\
& =0 \quad \text { for } x \geq b_{i},
\end{aligned}
$$

for all $i \in \mathbb{J}$. System (5.1) can be solved with the conditions

$$
\begin{aligned}
& V(0, i)=0, \\
& V\left(b_{i}-, j\right)=V\left(b_{i}+, j\right), \\
& V^{\prime}\left(b_{i}-, j\right)=V^{\prime}\left(b_{i}+, j\right), \\
& V^{\prime}\left(b_{i}-, i\right)=1, \\
& V^{\prime \prime}(\infty, i)=0,
\end{aligned}
$$

for all $i, j \in \mathbb{J}$. 


\subsection{The special case with two regimes}

In the special case with two regimes, the first method, i.e. iteration, is less efficient than solving the system of differential equations. So we consider the second method in this subsection.

Without loss of generality, let $0 \leq b_{1} \leq b_{2}$. To solve system (5.1), we have to consider the cases $x \in\left[0, b_{1}\right), x \in\left[b_{1}, b_{2}\right)$, and $x \in\left[b_{2}, \infty\right)$. Also, we need the following lemma. The proof is similar to Lemma 3.1 of [11] (see also [14]).

Lemma 5.1. Let $c_{1}$ and $c_{2}$ be two strictly positive constants. The system of equations

$$
0=\frac{\sigma_{1}^{2}}{2} r^{2}+\mu_{1} r-\left(c_{1}+q_{1}\right)+q_{1} s, \quad 0=\frac{\sigma_{2}^{2}}{2} r^{2}+\mu_{2} r-\left(c_{2}+q_{2}\right)+\frac{q_{2}}{s}
$$

on $(r, s)$ has four real roots $\left(r_{i}, s_{i}\right), i=1,2,3,4$, and $r_{1}<r_{2}<0<r_{3}<r_{4}$.

In the following, when we mention the roots of system (5.3), we assume that the $r_{i}$, $i=1,2,3,4$, are arranged as $r_{1}<r_{2}<0<r_{3}<r_{4}$.

If $x \in\left[0, b_{1}\right)$, system (5.1) yields

$$
\begin{aligned}
& 0=\frac{\sigma_{1}^{2}}{2} V^{\prime \prime}(x, 1)+\mu_{1} V^{\prime}(x, 1)-\left(\delta_{1}+q_{1}\right) V(x, 1)+q_{1} V(x, 2), \\
& 0=\frac{\sigma_{2}^{2}}{2} V^{\prime \prime}(x, 2)+\mu_{2} V^{\prime}(x, 2)-\left(\delta_{2}+q_{2}\right) V(x, 2)+q_{2} V(x, 1) .
\end{aligned}
$$

The solution of the above system of differential equation is given by

$$
\begin{aligned}
& V(x, 1)=A_{1} \mathrm{e}^{r_{1} x}+A_{2} \mathrm{e}^{r_{2} x}+A_{3} \mathrm{e}^{r_{3} x}+A_{4} \mathrm{e}^{r_{4} x}, \\
& V(x, 2)=A_{1} s_{1} \mathrm{e}^{r_{1} x}+A_{2} s_{2} \mathrm{e}^{r_{2} x}+A_{3} s_{3} \mathrm{e}^{r_{3} x}+A_{4} s_{4} \mathrm{e}^{r_{4} x},
\end{aligned}
$$

where $\left(r_{i}, s_{i}\right), i=1,2,3,4$, are the four roots of system (5.3) with $c_{i}=\delta_{i}, i=1,2$, and the $A_{i}, i=1,2,3,4$, are constants to be determined.

If $x \in\left[b_{1}, b_{2}\right)$, system (5.1) yields

$$
\begin{aligned}
0= & \frac{\sigma_{1}^{2}}{2} V^{\prime \prime}(x, 1)+\mu_{1} V^{\prime}(x, 1)-\left(\delta_{1}+\gamma_{1}+q_{1}\right) V(x, 1)+q_{1} V(x, 2) \\
& +\gamma_{1}\left[x-b_{1}+V\left(b_{1}, 1\right)\right], \\
0= & \frac{\sigma_{2}^{2}}{2} V^{\prime \prime}(x, 2)+\mu_{2} V^{\prime}(x, 2)-\left(\delta_{2}+q_{2}\right) V(x, 2)+q_{2} V(x, 1) .
\end{aligned}
$$

The solution of the above system is given by

$$
\begin{aligned}
& V(x, 1)=B_{1} \mathrm{e}^{\hat{r}_{1}\left(x-b_{1}\right)}+B_{2} \mathrm{e}^{\hat{r}_{2}\left(x-b_{1}\right)}+B_{3} \mathrm{e}^{\hat{r}_{3}\left(x-b_{1}\right)}+B_{4} \mathrm{e}^{\hat{r}_{4}\left(x-b_{1}\right)}+k_{1} x+l_{1}, \\
& V(x, 2)=B_{1} \hat{s}_{1} \mathrm{e}^{\hat{r}_{1}\left(x-b_{1}\right)}+B_{2} \hat{s}_{2} \mathrm{e}^{\hat{r}_{2}\left(x-b_{1}\right)}+B_{3} \hat{s}_{3} \mathrm{e}^{\hat{r}_{3}\left(x-b_{1}\right)}+B_{4} \hat{s}_{4} \mathrm{e}^{\hat{r}_{4}\left(x-b_{1}\right)}+k_{2} x+l_{2},
\end{aligned}
$$

where $\left(\hat{r}_{i}, \hat{s}_{i}\right), i=1,2,3,4$, are the four roots of system (5.3) with $c_{1}=\delta_{1}+\gamma_{1}$ and $c_{2}=\delta_{2}$, 
the $B_{i}, i=1,2,3,4$, are constants to be determined, and

$$
\begin{aligned}
k_{1} & =\frac{\left(q_{2}+\delta_{2}\right) \gamma_{1}}{\left(\gamma_{1}+q_{1}+\delta_{1}\right)\left(q_{2}+\delta_{2}\right)-q_{1} q_{2}}, \\
k_{2} & =\frac{q_{2} \gamma_{1}}{\left(\gamma_{1}+q_{1}+\delta_{1}\right)\left(q_{2}+\delta_{2}\right)-q_{1} q_{2}}, \\
l_{1} & =\frac{k_{1}}{\gamma_{1}}\left[k_{1} \mu_{1}+\frac{\gamma_{1}+q_{1}+\delta_{1}}{q_{2}} \mu_{2} k_{2}+\gamma_{1}\left(V\left(b_{1}, 1\right)-b_{1}\right)\right]-\frac{\mu_{2}}{q_{2}} k_{2}, \\
l_{2} & =\frac{k_{2}}{\gamma_{1}}\left[k_{1} \mu_{1}+\frac{\gamma_{1}+q_{1}+\delta_{1}}{q_{2}} \mu_{2} k_{2}+\gamma_{1}\left(V\left(b_{1}, 1\right)-b_{1}\right)\right] .
\end{aligned}
$$

If $x \in\left[b_{2}, \infty\right)$, system (5.1) yields

$$
\begin{aligned}
0= & \frac{\sigma_{1}^{2}}{2} V^{\prime \prime}(x, 1)+\mu_{1} V^{\prime}(x, 1)-\left(\delta_{1}+\gamma_{1}+q_{1}\right) V(x, 1)+q_{1} V(x, 2) \\
& +\gamma_{1}\left[x-b_{1}+V\left(b_{1}, 1\right)\right], \\
0= & \frac{\sigma_{2}^{2}}{2} V^{\prime \prime}(x, 2)+\mu_{2} V^{\prime}(x, 2)-\left(\delta_{2}+\gamma_{2}+q_{2}\right) V(x, 2)+q_{2} V(x, 1) \\
& +\gamma_{2}\left[x-b_{2}+V\left(b_{2}, 2\right)\right] .
\end{aligned}
$$

The solution of the above system is given by

$$
\begin{aligned}
& V(x, 1)=C_{1} \mathrm{e}^{\tilde{r}_{1}\left(x-b_{2}\right)}+C_{2} \mathrm{e}^{\tilde{r}_{2}\left(x-b_{2}\right)}+C_{3} \mathrm{e}^{\tilde{r}_{3}\left(x-b_{2}\right)}+C_{4} \mathrm{e}^{\tilde{r}_{4}\left(x-b_{2}\right)}+\tilde{k}_{1} x+\tilde{l}_{1}, \\
& V(x, 2)=C_{1} \tilde{s}_{1} \mathrm{e}^{\tilde{r}_{1}\left(x-b_{2}\right)}+C_{2} \tilde{s}_{2} \mathrm{e}^{\tilde{r}_{2}\left(x-b_{2}\right)}+C_{3} \tilde{s}_{3} \mathrm{e}^{\tilde{r}_{3}\left(x-b_{2}\right)}+C_{4} \tilde{s}_{4} \mathrm{e}^{\tilde{r}_{4}\left(x-b_{2}\right)}+\tilde{k}_{2} x+\tilde{l}_{2},
\end{aligned}
$$

where $\left(\tilde{r}_{i}, \tilde{s}_{i}\right), i=1,2,3,4$, are the four roots of system (5.3) with $c_{i}=\gamma_{i}+\delta_{i}, i=1,2$, the $C_{i}, i=1,2,3,4$, are constants to be determined, and

$$
\begin{aligned}
& \tilde{k}_{1}=\frac{q_{1} \gamma_{2}+\gamma_{1}\left(\gamma_{2}+q_{2}+\delta_{2}\right)}{\left(\gamma_{1}+q_{1}+\delta_{1}\right)\left(\gamma_{2}+q_{2}+\delta_{2}\right)-q_{1} q_{2}}, \\
& \tilde{k}_{2}=\frac{q_{2} \gamma_{1}+\gamma_{2}\left(\gamma_{1}+q_{1}+\delta_{1}\right)}{\left(\gamma_{1}+q_{1}+\delta_{1}\right)\left(\gamma_{2}+q_{2}+\delta_{2}\right)-q_{1} q_{2}}, \\
& \tilde{l}_{1}=\frac{q_{1} \mu_{2} \tilde{k}_{2}+\left(\gamma_{2}+q_{2}+\delta_{2}\right) \mu_{1} \tilde{k}_{1}+q_{1} \gamma_{2}\left(V\left(b_{2}, 2\right)-b_{2}\right)+\gamma_{1}\left(\gamma_{2}+q_{2}+\delta_{2}\right)\left(V\left(b_{1}, 1\right)-b_{1}\right)}{\left(\gamma_{1}+q_{1}+\delta_{1}\right)\left(\gamma_{2}+q_{2}+\delta_{2}\right)-q_{1} q_{2}}, \\
& \tilde{l}_{2}=\frac{q_{2} \mu_{1} \tilde{k}_{1}+\left(\gamma_{1}+q_{1}+\delta_{1}\right) \mu_{2} \tilde{k}_{2}+q_{2} \gamma_{1}\left(V\left(b_{1}, 1\right)-b_{1}\right)+\gamma_{2}\left(\gamma_{1}+q_{1}+\delta_{1}\right)\left(V\left(b_{2}, 2\right)-b_{2}\right)}{\left(\gamma_{1}+q_{1}+\delta_{1}\right)\left(\gamma_{2}+q_{2}+\delta_{2}\right)-q_{1} q_{2}} .
\end{aligned}
$$

The constants $A_{i}, B_{i}, C_{i}, i=1,2,3,4$, and the barrier levels $b_{1}$ and $b_{2}$ can be obtained from the conditions in (5.2).

Example 5.1. We choose all the parameters except $\gamma_{i}$ as in [8], namely,

$$
\begin{array}{llll}
\mu_{1}=0.06, & \sigma_{1}=0.24, & q_{1}=2, & \delta_{1}=0.04, \\
\mu_{2}=0.08, & \sigma_{2}=0.30, & q_{2}=3, & \delta_{2}=0.05 .
\end{array}
$$

Using the FindRoot function in MATHEMATICA ${ }^{\circledR}$, we calculated the optimal barrier levels for different $\gamma_{i}, i=1,2$. The results are given in Table 1. (When $\gamma_{2}=10$ and $\gamma_{1}=$ $50, \ldots, 500$, the results show that $b_{1}>b_{2}$, so we do not list them here.) The value (1.050, 1.070) for $\gamma_{1}=\gamma_{2}=\infty$ is taken from [8]. We can see that both the optimal barrier levels monotonically increase when the $\gamma_{i}, i=1,2$, increase, and they convergence to the case with $\gamma_{1}=\gamma_{2}=\infty$. This is consistent with the arguments of [3, p. 50]. 
TABLE 1: The optimal $\left(b_{1}, b_{2}\right)$ for different $\gamma_{i}, i=1,2$.

\begin{tabular}{rcccccc}
\hline & \multicolumn{6}{c}{$\gamma_{1}$} \\
\cline { 2 - 7 }$\gamma_{2}$ & 10 & 50 & 100 & 200 & 500 & $\infty$ \\
\hline 10 & $(0.9959,1.0059)$ & - & - & - & - & - \\
50 & $(1.0062,1.0338)$ & $(1.0264,1.0405)$ & $(1.0323,1.0417)$ & $(1.0367,1.0422)$ & $(1.0408,1.0426)$ & - \\
100 & $(1.0081,1.0418)$ & $(1.0274,1.0480)$ & $(1.0333,1.0490)$ & $(1.0376,1.0496)$ & $(1.0417,1.0499)$ & - \\
200 & $(1.0090,1.0477)$ & $(1.0279,1.0535)$ & $(1.0337,1.0545)$ & $(1.0381,1.0551)$ & $(1.0421,1.0554)$ & - \\
500 & $(1.0096,1.0532)$ & $(1.0282,1.0586)$ & $(1.0340,1.0600)$ & $(1.0383,1.0602)$ & $(1.0424,1.0605)$ & - \\
$\infty$ & - & - & - & - & - & $(1.050,1.070)$ \\
\hline
\end{tabular}

\section{Appendix A}

In this appendix we show that (4.13) admits a root in $(0, \infty)$. Since $h^{\prime}(x)>0,(4.13)$ is equivalent to

$$
\Delta_{i}^{\prime}(b)\left[s_{i} \frac{\delta_{i}+q_{i}}{\theta_{i}} \frac{h_{i}(b)}{h_{i}^{\prime}(b)}-1\right]=\Delta_{i}(b)\left[s_{i} \frac{\delta_{i}+q_{i}}{\theta_{i}}-\frac{h_{i}^{\prime \prime}(b)}{h_{i}^{\prime}(b)}\right] .
$$

Let

$$
f(b)=\Delta_{i}^{\prime}(b)\left[s_{i} \frac{\delta_{i}+q_{i}}{\theta_{i}} \frac{h_{i}(b)}{h_{i}^{\prime}(b)}-1\right]-\Delta_{i}(b)\left[s_{i} \frac{\delta_{i}+q_{i}}{\theta_{i}}-\frac{h_{i}^{\prime \prime}(b)}{h_{i}^{\prime}(b)}\right] .
$$

Obviously, $f(b)$ is continuous. From Sections 7 and 8 of [3], we know that

$$
\frac{h_{i}^{\prime \prime}\left(b_{0}\right)}{h_{i}^{\prime}\left(b_{0}\right)}=s_{i} \frac{\delta_{i}+q_{i}}{\theta_{i}}
$$

where

$$
b_{0}=\frac{1}{\alpha_{i}-\beta_{i}} \ln \frac{\beta_{i}^{2}}{\alpha_{i}^{2}}+\frac{1}{\alpha_{i}-\beta_{i}} \ln \frac{r_{i}-\alpha_{i}}{r_{i}-\beta_{i}}>0 .
$$

Noting that the left-hand side of (A.1) is positive, $h_{i}^{\prime \prime}(b) / h_{i}^{\prime}(b)$ is increasing and $\Delta_{i}(b)<0$, we have $f(0)>0$. To estimate $f(\infty)$, we can write

$$
f(b)=F_{1}(b)-F_{2}(b)-F_{3}(b)-F_{4}(b),
$$

where

$$
\begin{aligned}
F_{1}(b)= & \frac{2\left(s_{i}\left(\delta_{i}+q_{i}\right) / \theta_{i}-\alpha_{i}\right)}{\sigma_{i}^{2}\left(\alpha_{i}-\beta_{i}\right)} \mathrm{e}^{\alpha_{i} b}\left[\left(s_{i} \frac{\delta_{i}+q_{i}}{\theta_{i}} \frac{h_{i}(b)}{h_{i}^{\prime}(b)}-1\right)-\frac{1}{\alpha_{i}}\left(s_{i} \frac{\delta_{i}+q_{i}}{\theta_{i}}-\frac{h_{i}^{\prime \prime}(b)}{h_{i}^{\prime}(b)}\right)\right] \\
& \times \int_{0}^{b} \mathrm{e}^{-\alpha_{i} y} \sum_{j \neq i} q_{i j} u^{\prime}(y, j) \mathrm{d} y, \\
F_{2}(b)= & \frac{2\left(s_{i}\left(\delta_{i}+q_{i}\right) / \theta_{i}-\beta_{i}\right)}{\sigma_{i}^{2}\left(\alpha_{i}-\beta_{i}\right)} \mathrm{e}^{\beta_{i} b}\left[\left(s_{i} \frac{\delta_{i}+q_{i}}{\theta_{i}} \frac{h_{i}(b)}{h_{i}^{\prime}(b)}-1\right)-\frac{1}{\beta_{i}}\left(s_{i} \frac{\delta_{i}+q_{i}}{\theta_{i}}-\frac{h_{i}^{\prime \prime}(b)}{h_{i}^{\prime}(b)}\right)\right] \\
& \times \int_{0}^{b} \mathrm{e}^{-\beta_{i} y} \sum_{j \neq i} q_{i j} u^{\prime}(y, j) \mathrm{d} y,
\end{aligned}
$$




$$
\begin{aligned}
F_{3}(b)= & \frac{2}{\sigma_{i}^{2}}\left[\left(s_{i} \frac{\delta_{i}+q_{i}}{\theta_{i}} \frac{h_{i}(b)}{h_{i}^{\prime}(b)}-1\right)-\frac{1}{r_{i}}\left(s_{i} \frac{\delta_{i}+q_{i}}{\theta_{i}}-\frac{h_{i}^{\prime \prime}(b)}{h_{i}^{\prime}(b)}\right)\right] \\
& \times \int_{b}^{\infty} \mathrm{e}^{r_{i}(b-y)} \sum_{j \neq i} q_{i j} u^{\prime}(y, j) \mathrm{d} y, \\
F_{4}(b)= & \left(s_{i} \frac{\mu_{i} a_{i}}{\theta_{i}}-a_{i}\right)\left(s_{i} \frac{\delta_{i}+q_{i}}{\theta_{i}}-\frac{h_{i}^{\prime \prime}(b)}{h_{i}^{\prime}(b)}\right) .
\end{aligned}
$$

Note that $h_{i}(b) / h_{i}^{\prime}(b) \rightarrow 1 / \alpha_{i}$ and $h_{i}^{\prime \prime}(b) / h_{i}^{\prime}(b) \rightarrow \alpha_{i}$ as $b \rightarrow \infty$. Since

$$
\int_{0}^{b} \mathrm{e}^{-\alpha_{i} y} \sum_{j \neq i} q_{i j} u^{\prime}(y, j) \mathrm{d} y \leq \frac{1}{\alpha_{i}} \sum_{j \neq i} q_{i j} u^{\prime}(0, j),
$$

by l'Hôpital's rule,

$$
\mathrm{e}^{\alpha_{i} b}\left[\left(s_{i} \frac{\delta_{i}+q_{i}}{\theta_{i}} \frac{h_{i}(b)}{h_{i}^{\prime}(b)}-1\right)-\frac{1}{\alpha_{i}}\left(s_{i} \frac{\delta_{i}+q_{i}}{\theta_{i}}-\frac{h_{i}^{\prime \prime}(b)}{h_{i}^{\prime}(b)}\right)\right] \rightarrow 0,
$$

so we have $F_{1}(b) \rightarrow 0$ as $b \rightarrow \infty$. Recalling that $\boldsymbol{u} \in \mathbb{D}$, we have

$$
\begin{aligned}
\mathrm{e}^{\beta_{i} b} \int_{0}^{b} \mathrm{e}^{-\beta_{i} y} \sum_{j \neq i} q_{i j} u^{\prime}(y, j) \mathrm{d} y & \leq \mathrm{e}^{\beta_{i} b}\left(\sum_{j \neq i} q_{i j} u(b, j)-\sum_{j \neq i} q_{i j} u(0, j)\right) \\
& \rightarrow 0 \quad \text { as } b \rightarrow \infty .
\end{aligned}
$$

Thus, $F_{2}(b) \rightarrow 0$ as $b \rightarrow \infty$. Recalling that

$$
\int_{b}^{\infty} \mathrm{e}^{r_{i}(b-y)} \sum_{j \neq i} q_{i j} u^{\prime}(y, j) \mathrm{d} y \rightarrow 0 \quad \text { as } b \rightarrow \infty
$$

we have $F_{3}(b) \rightarrow 0$ as $b \rightarrow \infty$. Since $h_{i}^{\prime \prime}(b) / h_{i}^{\prime}(b)$ is increasing and $s_{i} \mu_{i}-\theta_{i}=-\sigma_{i}^{2} s_{i}^{2} / 2<0$, we have

$$
F_{4}(b) \rightarrow\left(s_{i} \frac{\mu_{i} a_{i}}{\theta_{i}}-a_{i}\right)\left(s_{i} \frac{\delta_{i}+q_{i}}{\theta_{i}}-\alpha_{i}\right)>0 \quad \text { as } b \rightarrow \infty .
$$

Thus, we have

$$
f(b) \rightarrow-\left(s_{i} \frac{\mu_{i} a_{i}}{\theta_{i}}-a_{i}\right)\left(s_{i} \frac{\delta_{i}+q_{i}}{\theta_{i}}-\alpha_{i}\right)<0 \quad \text { as } b \rightarrow \infty .
$$

Then, by the continuity of $f(b),(4.13)$ has a root in $(0, \infty)$.

\section{Acknowledgements}

We would like to thank the anonymous referee for valuable comments and suggestions. This work was supported by the National Natural Science Foundation of China (grant number 10971068), the Doctoral Program Foundation of the Ministry of Education of China (grant number 20110076110004), the Program for New Century Excellent Talents in University (grant number NCET-09-0356), the Fundamental Research Funds for the Central Universities, the PhD Scholarship Fund of ECNU (grant number 2010050), the Ministry Reward for Excellent Doctors in Academics (grant number MXRZZ2010006), and the Research Grants Council of the Hong Kong Special Administrative Region, China (project number HKU 706209P). 


\section{References}

[1] Albrecher, H., BäUerle, N. And Thonhauser, S. (2011). Optimal dividend-payout in random discrete time. Statist. Risk Modelling 28, 251-276

[2] Albrecher, H., Cheung, E. C. K. And Thonhauser, S. (2011). Randomized observation periods for the compound poisson risk model: dividends. ASTIN Bull. 41, 645-672.

[3] Albrecher, H., Gerber, H. U. and Shiu, E. S. W. (2011). The optimal dividend barrier in the gamma-omega model. Europ. Actuarial J. 1, 43-55.

[4] Bäuerle, N. ANd Rieder, U. (2011). Markov Decision Processes with Applications to Finance. Springer, Heidelberg.

[5] Bayraktar, E. ANd LudKovski, M. (2009). Sequential tracking of a hidden Markov chain using point process observations. Stoch. Process. Appl. 119, 1792-1822.

[6] Davis, M. H. A. (1993). Markov Models and Optimization. Chapman \& Hall, London.

[7] De Finetti, B. (1957). Su un'impostazione alternativa della teoria collecttiva del rischio. In Trans. 15th Internat. Congress of Actuaries, pp. 433-443.

[8] Jiang, Z. ANd Pistorius, M. (2012). Optimal dividend distribution under Markov-regime switching. To appear in Finance Stoch.

[9] Kyprianou, A. E. (2006). Introductory Lectures on Fluctuations of Lévy Processes with Applications. Springer, Berlin.

[10] Polyanin, A. D. and Zaitsev, V. F. (1995). Handbook of Exact Solutions for Ordinary Differential Equations. CRC Press, Boca Raton, FL.

[11] Sotomayor, L. R. And CADEnillas, A. (2011). Classical and singular stochastic control for the optimal dividend policy when there is regime switching. Insurance Math. Econom. 48, 344-354.

[12] Wang, H. (2001). Some control problems with random intervention times. Adv. Appl. Prob. 33, 404-422.

[13] WeI, J. AND WANG, R. (2012). A note on the optimal dividend strategies under the gamma-omega risk model. Submitted.

[14] WeI, J., YANG, H. AND WANG, R. (2011). Optimal threshold dividend strategies under the compound Poisson model with regime switching. In Stochastic Analysis with Financial Applications, eds N. P. A. Kohatsu-Higa and S. Sheu, Birkhäser, Basel, pp. 413-429. 